\title{
Reprogramming of leukemic cell metabolism through the naphthoquinonic compound Quambalarine B
}

\author{
Karel Vališ ${ }^{1,2}$, Valéria Grobárová ${ }^{3}$, Lucie Hernychová1,3, Martina Bugáňováa,5, Daniel \\ Kavan ${ }^{2,4}$, Martin Kalous ${ }^{3}$, Jiří Černý ${ }^{6}$, Eva Stodůlková ${ }^{4}$, Marek Kuzma ${ }^{4}$, Miroslav \\ Flieger ${ }^{4}$, Jan Černý ${ }^{3}$ and Petr Novák ${ }^{1,2}$ \\ ${ }^{1}$ BIOCEV, Institute of Microbiology, v.v.i., The Czech Academy of Sciences, Vestec, Czech Republic \\ ${ }^{2}$ Department of Biochemistry, Faculty of Science, Charles University, Prague, Czech Republic \\ ${ }^{3}$ Department of Cell Biology, Faculty of Science, Charles University, Prague, Czech Republic \\ ${ }^{4}$ Institute of Microbiology, v.v.i., The Czech Academy of Sciences, Prague, Czech Republic \\ ${ }^{5}$ Faculty of Chemical Technology, University of Chemistry and Technology, Prague, Czech Republic \\ ${ }^{6}$ BIOCEV, Institute of Biotechnology, v.v.i., The Czech Academy of Sciences, Vestec, Czech Republic \\ Correspondence to: Karel Vališ, email: karel.valis@biomed.cas.cz \\ Petr Novák, email: pnovak@biomed.cas.cz
}

Keywords: metabolism, leukemia, naphthoquinones, mitochondria, therapy

Received: July 14, $2017 \quad$ Accepted: September 21, $2017 \quad$ Published: October 07, 2017

Copyright: Vališ et al. This is an open-access article distributed under the terms of the Creative Commons Attribution License 3.0 (CC BY 3.0), which permits unrestricted use, distribution, and reproduction in any medium, provided the original author and source are credited.

\section{ABSTRACT}

Abnormalities in cancer metabolism represent potential targets for cancer therapy. We have recently identified a natural compound Quambalarine B (QB), which inhibits proliferation of several leukemic cell lines followed by cell death. We have predicted ubiquinone binding sites of mitochondrial respiratory complexes as potential molecular targets of QB in leukemia cells. Hence, we tracked the effect of QB on leukemia metabolism by applying several omics and biochemical techniques. We have confirmed the inhibition of respiratory complexes by QB and found an increase in the intracellular AMP levels together with respiratory substrates. Inhibition of mitochondrial respiration by QB triggered reprogramming of leukemic cell metabolism involving disproportions in glycolytic flux, inhibition of proteins 0 -glycosylation, stimulation of glycine synthesis pathway, and pyruvate kinase activity, followed by an increase in pyruvate and a decrease in lactate levels. Inhibition of mitochondrial complex I by QB suppressed folate metabolism as determined by a decrease in formate production. We have also observed an increase in cellular levels of several amino acids except for aspartate, indicating the dependence of Jurkat (T-ALL) cells on aspartate synthesis. These results indicate blockade of mitochondrial complex I and II activity by QB and reduction in aspartate and folate metabolism as therapeutic targets in T-ALL cells. Anti-cancer activity of QB was also confirmed during in vivo studies, suggesting the therapeutic potential of this natural compound.

\section{INTRODUCTION}

Cancer cells represent rapidly dividing population of cells with a number of different metabolic requirements, which exemplify potential targets for cancer therapy. Alterations in cancer cell metabolism were observed for the first time by German biochemist Otto Warburg in 1920s. He described that cancer cells rely on glycolytic metabolism even in the presence of oxygen and produce lactate as glycolytic end product [1]. Based on these observations, Warburg hypothesized that cancer cells carry dysfunctional mitochondria [2]. On the other hand, under physiological conditions, some proliferating cell types were found to preferentially rely on glucose metabolism without any mitochondrial defect [3].

Pyruvate, an end product of glycolytic pathway, mainly supplies mitochondrial metabolism. Importantly, metabolic pathways derived from glycolysis also 
provide intermediates for the synthesis of amino sugars, amino acids, and nucleotides. Recent reports point out decreased activity of pyruvate kinases (PKM) in cancer cells due to preferential expression of PKM2 isoform driven by C-MYC oncoprotein and inhibition of pyruvate dehydrogenase $(\mathrm{PDH})$ activity, as a result of inhibitory phosphorylation catalyzed by PDH kinase (PDK) [4, 5]. These events may lead to inhibition of mitochondrial complex I activity in the cancer cells. PKM2 exists in cancer cells as a dimer with lower catalytic activity, which results in the accumulation of glycolytic intermediates and their subsequent transformation in biosynthetic pathways [6]. Hence, increased glycolysis contributes to the biosynthesis of building blocks indispensable for the proliferation of cancer cells. Biosynthetic pathways utilizing glycolytic intermediates involve hexosamine synthetic pathway, pentose pathway, and serine/glycine synthetic pathway, which regulates folate metabolism and is involved in purine synthesis and mitochondrial metabolism [7]. Consistently with these findings, activators of PKM2 activity act as tumor suppressors [8]. Recent studies suggest an important role of mitochondria in cancer cell proliferation due to the synthesis of aspartate from oxaloacetate and dependency of cancer cells on glutamine supplementation [9-11]. In this logic, cancer cells utilize conversion of glutamine to alpha-ketoglutarate (AKG) as a major source of metabolites, which feed oxaloacetate synthesis and act as a source of electrons for electron transport chain (ETC) orchestrated by the complex II activity. This switch in cancer cell metabolism is also linked to C-MYC activity. Several signaling pathways were recently identified as regulators of C-MYC expression and cancer cell metabolism. These pathways involve AMPK and Hippo signaling pathways, which can act as potent tumor suppressors $[12,13]$.

Biosynthetic pathways represent a promising target for cancer therapy. Purine synthesis pathway is one of the first metabolic pathways targeted for cancer therapy. Purine synthesis can be inhibited using compounds interfering with folate metabolism (anti-folate), which represents an important cofactor in purine synthesis [14]. Another example of pioneer cancer therapy targeting metabolism is depletion of asparagine (and glutamine) using asparaginase. This therapy utilizes dependency of several cancer cell types on asparagine and glutamine and thus, inhibits cancer cells proliferation followed by cancer cell death. On the other hand, asparaginase treatment triggers reprogramming of cancer cell metabolism toward fatty acid oxidation, providing the support of tricarboxylic acid (TCA) cycle intermediates. Consistent with these findings, the inhibitors of fatty acid oxidation leads to an increase in the sensitivity of cancer cells to asparaginase treatment [15]. Other metabolic vulnerabilities of cancer cells are reviewed by Vander Heiden [16].
Quinones represent natural aromatic compounds participating in key metabolic processes nearly in all living organisms and can be exemplified by ubiquinone (UbQ). This molecule acts as a key electron transporter during several biochemical reactions involved in cellular energetic and biosynthetic pathways. UbQ is structurally related to naphthoquinones, which are synthesized in a large spectrum of organisms involving bacteria, plants, and fungi. Moreover, 1,4-naphthoquinones (NQ) represent an important group of anti-cancer drugs commonly used in clinical practice, mainly acting as redox cyclers and alkylating agents [17]. The oxidoreduction of quinone induces reactive oxygen species (ROS) production followed by cancer cell death [18]. NQ can be reduced by several NADH or NADPH oxidoreductases and can also interfere with their specific functions. Several NQs were described as potent inhibitors of mitochondrial respiratory complexes disturbing the energetic and metabolic balance of the cell [19-21]. NQ carrying non-substituted C2 or C3 position may react with thiols or amines of biomolecules to form covalent adducts. These NQ-based covalent modifications often interfere with proper functioning of target molecules as in the case of protein tyrosine phosphatase inhibition triggered by covalent modification of active cysteine residue [22].

We have recently identified a group of novel and natural naphthoquinonic compounds with potential anticancer activity and confirmed anti-leukemic activity of the novel compound 1,4-naphthoquinone Quambalarine B (QB). QB effectively blocks proliferation of several leukemic cell lines and induces loss of proton gradient across the inner mitochondrial membrane [23]. QB also inhibits expression of C-MYC oncoprotein and attenuates mTOR signaling in several tested leukemic cell lines [24]. Since QB possesses substituted C2 and C3 positions in its structure, we presume redox cycling together with target oxidoreductases interference as a key mechanism responsible for QB anti-cancer activity. Hence, we employed several proteomic and metabolomic techniques to uncover the molecular mechanism behind the QB effect on the metabolism of leukemic cells. We suggest a model of metabolic reprogramming, which involves inhibition of mitochondrial respiration, accumulation or inhibition of specific metabolites and amino acids, and imbalance in the regulation of glycolysis and glycolysis-derived biosynthetic pathways. QB-driven rearrangement of leukemic metabolism leads to inhibition of proliferation partially through the simulation of nutrient starvation. Importantly, we confirmed QB anti-tumor activity in vivo using mouse xenograft model verifying QB as a new promising anti-cancer drug. Finally, our model provides novel and complex insight into the metabolism regulatory network in leukemic cells and highlights the novel metabolic circuits representing new promising targets for leukemia treatment. 


\section{RESULTS}

\section{QB acts as a competitive inhibitor of ubiquinone binding on complex I and complex II}

Reflecting structural similarity, we focused on a key electron transporter UbQ as a possible target to reveal the molecular mechanism behind the QB effects on mitochondrial function. We predicted binding mode for UbQ and QB docking to mitochondrial respiratory $\mathrm{CI}$ and CII structures using molecular modeling strategy. For the CII structure, where the UbQ position was known from crystal structures, we could compare the performance of used docking algorithm. The crystal orientation was reproduced by the second pose (with the predicted binding affinity for the slightly tilted first pose being only $0.1 \mathrm{kcal} / \mathrm{mol}$ more favorable). The predicted QB binding site overlapped with the UbQ position and its binding was more favorable by about $0.5 \mathrm{kcal} / \mathrm{mol}$. In the case of CI, both ligands shared a binding site within the expected UbQ binding cavity, and again QB binding affinity was more favorable compared to UbQ by about $0.5 \mathrm{kcal} / \mathrm{mol}$. This suggests that QB may affect UbQ interactions with respect to CI and CII structures (Figure 1A), being the higher affinity interactor.

\section{QB treatment inhibits activity of mitochondrial complexes I and II}

To validate the possible competitive inhibitory effect of QB on the activity of mitochondrial respiratory complexes in vivo, the mitochondria isolated from rat hepatocytes were used as a model system, and oxygraphy was used as an experimental approach. We observed significant inhibition of complexes I and II activity during QB incubation with isolated mitochondria compared to mitochondria incubated with DMSO as a control, suggesting a possible UbQ competitive mechanism underlying QB bioactivity (Figure 1B). The respiratory ratio was significantly lower in mitochondria incubated with $20 \mu \mathrm{mol} / \mathrm{L}$ QB (1.3) compared to control (4.28). To continue to the whole cell system, we estimated the inhibitory concentration $\left(\mathrm{IC}_{50}\right)$ of $\mathrm{QB}$ toward oxidoreductases in leukemia cells by MTS assay. Using this method, we calculated $\mathrm{IC}_{50}=2 \mu \mathrm{mol} / \mathrm{L}$, and this concentration of QB was used in all following experiments (Supplementary Figure 1). Inhibition of individual respiratory complexes often resulted in the accumulation of input metabolites, i.e., pyruvate and succinate [10, 25]. We confirmed increased levels of both metabolites in leukemia cells after QB treatment using NMR and enzymatic techniques, respectively (Figure 1C and 1D). Moreover, at least two other metabolic pathways are known to drive pyruvate transformation in cancer cells. Pyruvate can be reduced to lactate by the cytosolic lactate dehydrogenase (LDH) activity or transaminated into alanine by mitochondrial alanine transaminase activity, which also produces AKG from glutamate. We tracked pyruvate metabolic pathway after QB treatment on the basis of alanine level estimation using NMR, enzymatic and HPLC techniques. We confirmed significant changes in alanine and lactate concentrations. We detected significant increase for intracellular alanine as well as for alanine in the culture medium, suggesting its transport out of the cells (Figure 1E and 1F). On the other hand, the intracellular concentration of lactate remained constant (data not shown), but it decreased extracellularly, suggesting inhibition of lactate synthesis and transport after QB treatment (Figure 1G). Finally, we determined cellular ATP and AMP concentrations after QB treatment using NMR analysis. We observed a slight decrease in signal common to all adenosine nucleotides, but on the other hand, the strong intensity was found in the signal belonging solely to AMP. These results clearly showed an increase in AMP fraction from all adenosine nucleotide variants $(\mathrm{AMP}+\mathrm{ADP}+\mathrm{ATP}$; Figure $1 \mathrm{H}$ and $1 \mathrm{I})$. These observations can play an important role in global QBdriven reprogramming of cancer metabolism since lactatedriven acidification of cytosol and alanine levels represent well-known regulators of glycolytic flux.

\section{QB treatment has insignificant effect on transport of glucose into leukemia cells}

To clarify possible changes in leukemia metabolism triggered by QB treatment, we focused on glycolytic pathway due to its prime role in cancer cell proliferation. As a first step, we quantified uptake of glucose during QB treatment that showed a decrease in the glucose levels in culture cell medium using enzymatic assay. We detected an insignificant increase in glucose uptake after QB treatment (Figure 2A). Considering that GLUT1 represents major glucose transporter in Jurkat cells, we tested the effects of QB on GLUT1 mRNA and protein levels using RTqPCR, immunoblotting and LFQ analysis. We observed a significant decrease in GLUT1 mRNA levels and a slight increase in GLUT1 protein levels, which suggests potential stabilization of the GLUT1 protein on the plasma membrane (Figure 2B-2D).

\section{QB shows multiple effects on the glycolytic pathway functions}

To characterize the effect of QB on the levels of individual enzymes of the glycolytic pathway, we analyzed LFQ data and quantified relative levels of individual glycolytic enzymes. We also quantified the levels of all glycolytic enzymes (except phosphoglucose isomerase and enolase). After QB treatment, the level of hexokinase (HK1) was not significantly increased, but the levels of phosphofructokinase (PFK), glyceraldehyde 3-phosphate dehydrogenase (GAPDH), phosphoglycerate 
mutase (PGM1), and pyruvate kinase M (PKM) were significantly elevated (Figure 2E). This result suggests an increased rate of glycolytic flux triggered by QB in leukemia cells. PFK catalyzes the irreversible conversion of fructose 6-phosphate to fructose 1,6-bisphosphate and

A

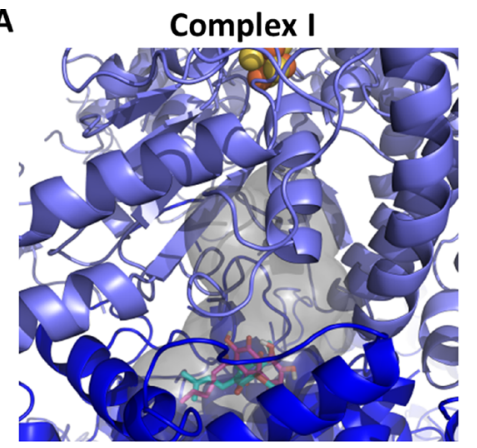

\section{B}

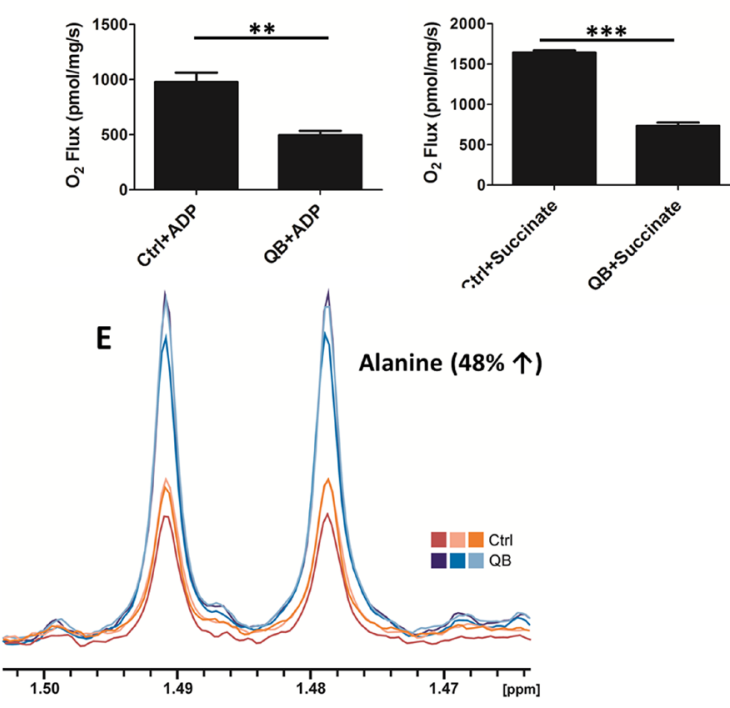

$\mathbf{F}$

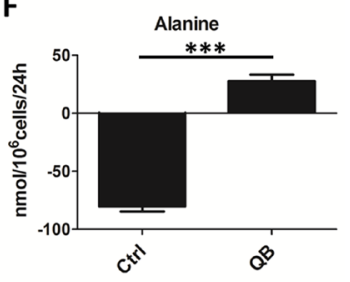

G

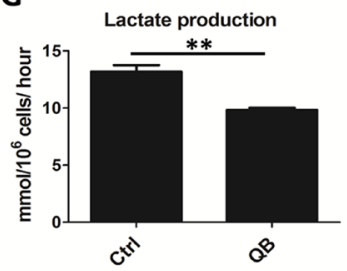

represents one of the key regulatory glycolytic enzymes. Additionally, fructose 6-phosphate represents the substrate for glutamine--fructose 6-phosphate amidotransferase (GFAT), which acts as the rate-limiting enzyme in UDPacetylglucosamine (UDP-GlcNAc) synthesis pathway that

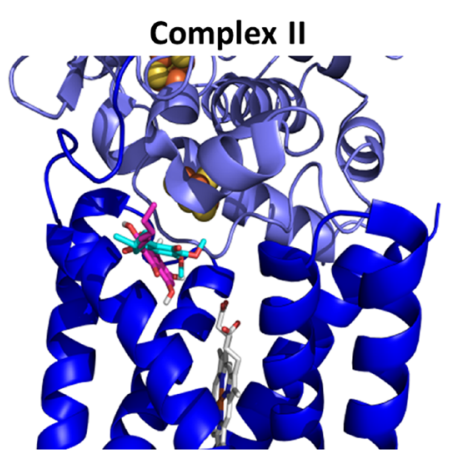

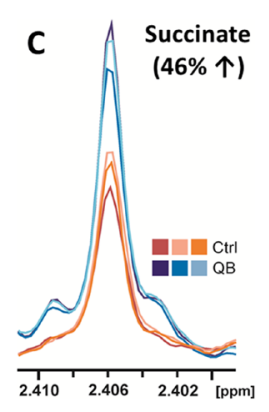
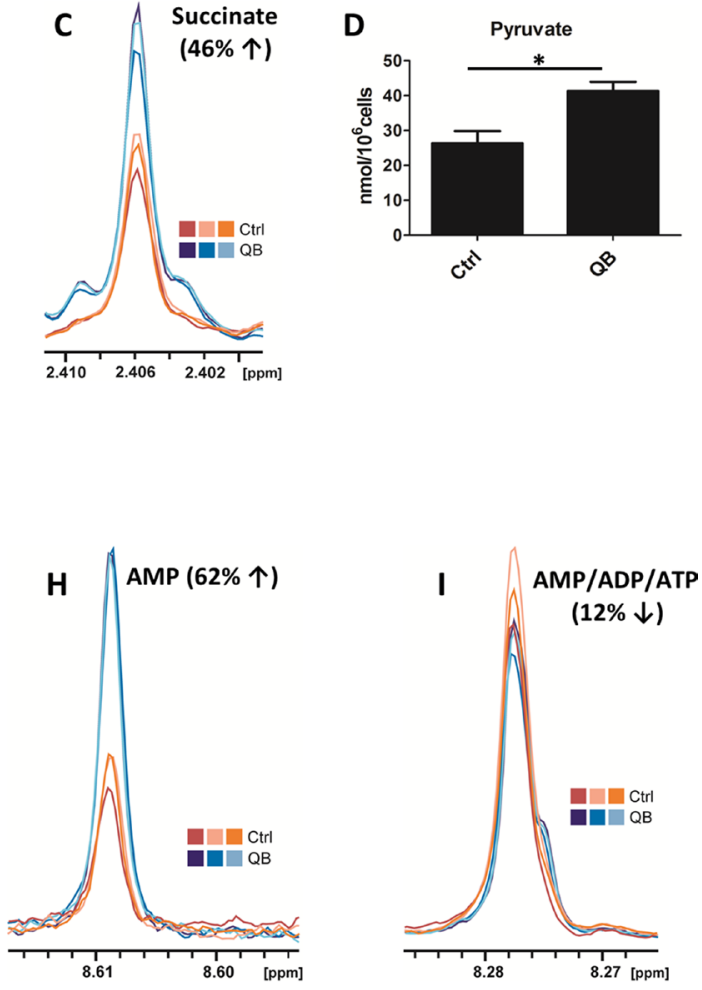

Figure 1: Quambalarine B (QB) inhibits the activity of mitochondrial complexes I and II in Jurkat cells. (A) Molecular docking of QB to ubiquinone binding site in mitochondrial complex I and complex II. The grey surface corresponds to a potential ubiquinone binding cavity as obtained by analysis of the crystal structure by $3 \mathrm{~V}$ program. (B) Effect of QB $(20 \mu \mathrm{mol} / \mathrm{L})$ on the activity of individual mitochondrial complexes determined by oxygen consumption. Oxygen uptake in isolated rat mitochondria is expressed as pmol/s/mg protein. (C) Levels of succinate in control (orange lines) and QB-treated (blue lines) cells determined using NMR analysis. (D) Levels of pyruvate in control (ctrl) and QB-treated cells (QB) determined by enzymatic assay. (E) Levels of intracellular alanine in control (orange lines) and QB-treated cells (blue lines) determined by NMR analysis. (F) Changes in alanine levels in the culture medium of control (ctrl) and QB-treated cells (QB) after $24 \mathrm{~h}$ of incubation determined by HPLC analysis. (G) Lactate production by control (ctrl) and QB-treated cells (QB) determined by enzymatic assay. (H) AMP levels in control (orange lines) and QB-treated (blue lines) cells determined by NMR analysis. (I) AMP/ADP/ATP levels in control (orange lines) and QB-treated (blue lines) cells determined by NMR analysis. Data are shown as means from three independent experiments \pm SEM. ${ }^{*}$, significant differences with $P \leq 0.05$. ${ }^{* *}$, significant differences with $P \leq 0.01$. ${ }^{* * *}$, significant differences with $P \leq 0.001$. 
is indispensable for proper $\mathrm{N}$ - and $\mathrm{O}$ - protein glycosylation [26]. Hence, we quantified fructose 6-phosphate levels using enzymatic assay after QB treatment and observed a significant decrease (approx. 8-fold) in its level in cells treated with QB (Figure 2F). Since glucose uptake slightly increased after QB treatment, we presumed that low level of the fructose 6-phosphate was a result of increased PFK activity (GFAT represents enzyme with low-affinity to fructose 6-phosphate utilizing approx. 5\% of the total glucose in the cell). PFK activity has been reported to be negatively regulated by ATP level, O-GlcNAcylation and low cytosolic $\mathrm{pH}$ (result of increased lactate concentration) and positively regulated by AMP levels (AMP diminished effect of ATP and $\mathrm{pH}$ ) which were increased after QB treatment (Figure 1H and 1I) [27].

\section{QB treatment inhibits protein O-glycosylation in leukemic cells}

Considering the effect on fructose 6-phosphate utilization, we focused on the determination of GFAT enzyme level. We detected an insignificant increase in levels of GFAT after QB treatment using LFQ analysis (data not shown). Hence, we monitored the effect of QB on
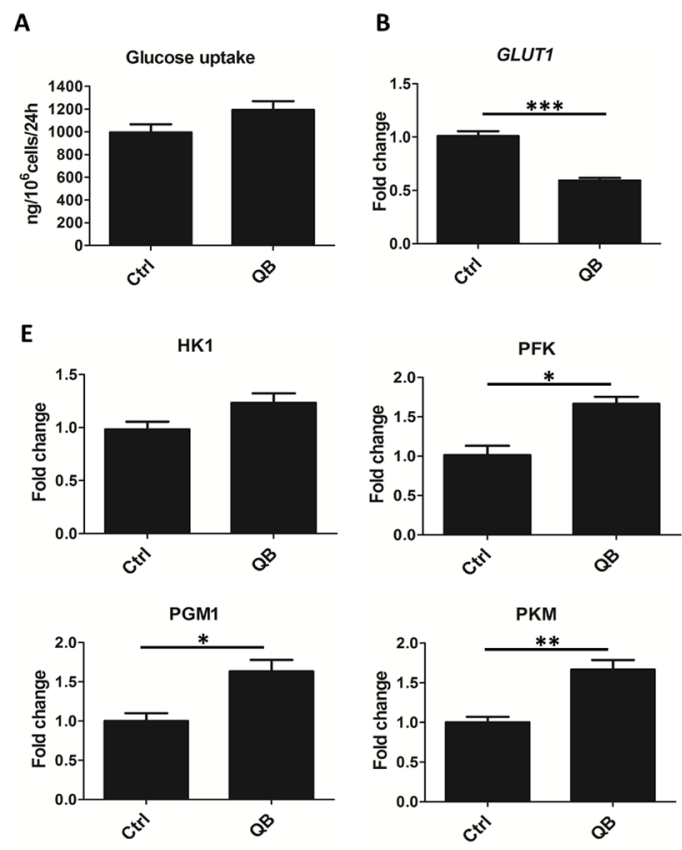

protein glycosylation. Using immunoblotting, we observed nearly total abrogation of protein O-glycosylation after QB treatment (Figure 2G). These results suggest inhibition of protein O-glycosylation, probably due to low levels of fructose 6-phosphate, the first substrate in hexosamine synthetic pathway. Moreover, the absence of protein O-glycosylation possessed a strong effect on cellular oncogenic signaling, adhesion, proliferation and survival [28]. Several reports have highlighted the role of $\mathrm{O}$-glycosylation in the stabilization of crucial oncogenic transcription factors (e.g. C-MYC) and pro-survival signaling pathways (e.g. PI3K) [29, 30]. It was also shown that O-GlcNAc modification of PFK molecule inhibited its enzyme activity, thereby, increasing glucose flux through the pentose pathway supporting cancer cells proliferation $[26,27]$.

\section{QB shows strong impact on 3PG-serine- glycine-5,10CH2-THF-10CHO-THF-formate biosynthetic pathway}

Recently, novel metabolic pathway feeding mitochondrial respiratory complex I was decrypted. This pathway originated from serine-glycine synthesis

C

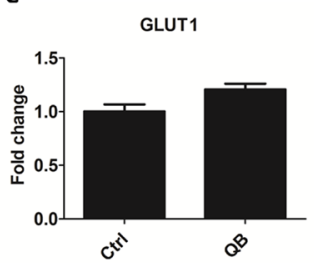

D
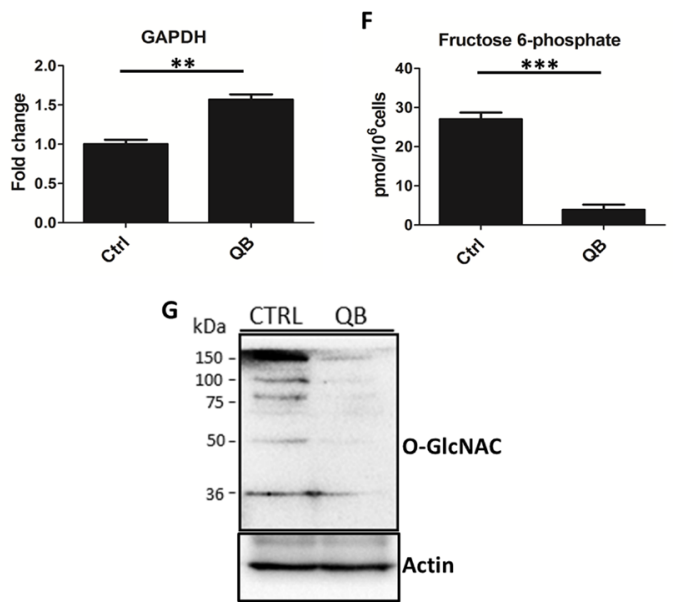

Figure 2: Quambalarine B (QB) treatment has a strong impact on glycolysis. (A) Changes in glucose levels in culture medium were determined using enzymatic assay. (B) Relative levels of GLUT1 mRNA in QB-treated cells compared to control (ctrl) were determined using RT-qPCR method. (C) Relative levels of GLUT1 protein in QB-treated cells compared to control (ctrl) quantified by LFQ analysis. (D) GLUT1 protein levels in QB-treated cells compared to control (ctrl) determined by immunoblot. (E) Relative levels of glycolytic enzymes, hexokinase (HK1), phosphofructokinase (PFK), glyceraldehyde 3-phosphate dehydrogenase (GAPDH), phosphoglycerate mutase (PGM1), and pyruvate kinase M (PKM) in QB-treated cells compared to control (ctrl) determined by LFQ analysis. (F) Fructose 6-phosphate levels in control (ctrl) and QB-treated cells (QB) determined by enzymatic assay. (G) Levels of O-GlcNAC modifications of individual proteins determined by immunoblot comparing treated (QB) and control (CTRL) cells. Data are shown as means from three independent experiments \pm SEM. ${ }^{*}$, significant differences with $P \leq 0.05 .{ }^{* *}$, significant differences with $P \leq 0.01{ }^{* * *}$, significant differences with $P \leq 0.001$. The images shown are representative of three independent experiments. In all experiments cells were treated with $2 \mu \mathrm{mol} / \mathrm{L}$ of QB. 
pathway. In this pathway, glycolytic intermediate 3-phosphoglycerate feeds serine synthesis, which is converted in mitochondria to glycine by the activity of serine hydroxymethyltransferase (SHMT2) while producing 5,10-methylene-tetrahydrofolate $(5,10-\mathrm{CH} 2-$ THF). Glycine can be subsequently utilized for protein and glutathione synthesis or can be transported out of the cell. Since a high concentration of glycine is considered highly cytotoxic, cellular level of glycine must be tightly regulated [31]. 5,10-CH2-THF is subsequently oxidized to 10 -formyl-tetrahydrofolate $(10-\mathrm{CHO}-\mathrm{THF})$ by the activity of 5,10-methylene-tetrahydrofolate dehydrogenase (MTHFD2), which produces NADH that can serve as an electron donor to mitochondrial complex I. 10-formyltetrahydrofolate is then cleaved to THF and formate by the activity of reverse 10-formyl-tetrahydrofolate synthase generating one molecule of ATP from ADP [7].

To uncover the effect of $\mathrm{QB}$ treatment on the glycine synthesis pathway, we quantified the levels of individual enzymes using LFQ analysis. We observed a significant increase in the levels of D-3-phosphoglycerate dehydrogenase (PGDH) (268\% of non-treated control) and phosphoserine aminotransferase (PSAT) (223\% of non-treated control), but no significant changes were observed in SHMT2 and MTHFD2 levels (data not shown) after QB treatment (Figure 3A). Then we determined the effect of $\mathrm{QB}$ on intracellular serine levels and uptake of serine from the culture medium using NMR and HPLC analysis. NMR analysis uncovered an increase in intracellular levels of serine (Figure 3B). Unfortunately, this result cannot be clearly confirmed by HPLC analysis due to the identical retention time of serine and asparagine. However, we observed a significant decrease in serine/asparagine uptake (approx. $50 \%$ of non-treated control) in leukemic cells after QB treatment (Figure 3C). Using the same setup, we determined the levels of glycine and glycine uptake. We detected slight increase in the intracellular glycine levels after QB treatment using NMR analysis (data not shown), which probably reflected tight control of glycine concentration in cancer cells [31]. Surprisingly, we did not detect any significant uptake of glycine by control leukemic cells; however, we observed a significant increase in extracellular glycine levels during QB treatment using HPLC analysis, suggesting export of glycine out of the cell (Figure 3D). We also determined an increase in the glutathione (GSH) levels after QB treatment, which represented another possible route in the glycine metabolism [7] (Figure 3E). These results indicated that synthesis of glycine from glycolytic intermediate 3-phosphoglycerate can represent a major source of glycine in leukemic cells pointing to the exclusive role of glycolysis in leukemic cells proliferation. Then, we tracked the effect of QB on formate production employing NMR analysis and observed a decrease in the intracellular formate levels (Figure 3F). It is known that the rate of formate production depends on complex I activity since metformin and rotenone (inhibitors of complex I) inhibit formate production in several cancer cell lines [7, 32]. Mitochondria-derived formate represents an important intermediate in the cytosolic synthesis of purines or can be exported out of the cell. Hence, our observations provided another evidence of complex I blockade during QB treatment. Moreover, the high levels of formate and lactate production in nontreated cells suggest involvement of the folate pathway in mitochondrial metabolism.

\section{QB increases PKM activity in leukemic cells}

PKM activity represents another important regulator of glycolytic flux. It is well known that cancer cells predominantly express PKM2 isoform, which possesses low enzymatic activity as a result of C-MYC oncogenic activity [4]. PKM2 expression triggers the accumulation of upper glycolytic intermediates, which can feed biosynthetic pathways derived from glycolysis [33]. On the other hand, serine represents one of the end-point glycolysis-derived metabolites and allosterically activates PKM2 activity [34]. We have previously demonstrated that QB treatment decreases C-MYC protein levels and PKM2 mRNA levels in several leukemic cells [24]. In this study, we also demonstrated an increase in the serine levels after QB treatment; hence, we tested PKM activity after QB treatment using enzymatic assay. We confirmed a significant increase in PKM activity triggered by QB treatment along with increased levels of intracellular pyruvate (Figures 3G and 1D). Several other compounds activating PKM activity in cancer cells were recently tested as potential anti-cancer drugs. These compounds impaired anabolic metabolism and inhibited cancer cell proliferation, demonstrating the important role of glycolysis-derived pathways in cancer cells proliferation and survival [35].

\section{Glucose starvation protects leukemic cells against cell death induced by $Q B$}

We tested the effect of glucose starvation to highlight the importance of glucose metabolism in QB anti-cancer activity. Cells were pre-incubated for $24 \mathrm{~h}$ in RPMI1640 medium without glucose supplemented with FBS followed by treatment with $\mathrm{EC}_{50}$ concentration of QB $(8 \mu \mathrm{mol} / \mathrm{L})$ for $24 \mathrm{~h}$. Fractions of live/dead cells were determined using FACS analysis. Surprisingly, we detected significantly reduced fractions of death cells after glucose starvation against cells grown in standard cultivation medium (Figure $3 \mathrm{H}$ ). This result suggests protecting effect of adaptation to glucose starvation against cell death induced by QB. 


\section{QB treatment inhibits glutamine metabolism and aspartate synthesis in leukemia cells}

We tested the effect of QB on glutamine metabolism and subsequent oxaloacetate and aspartate synthesis. We quantified the expression of SLC1A5 glutamine transporter using LFQ analysis and immunoblotting, and both the techniques detected a slight increase in SLC1A5 levels after QB treatment (Figure 4A, 4B). Using LFQ analysis we also observed increase by 1.3 and 1.5 folds for other potential glutamine transporters represented by SLC38A1 and SLC38A2, respectively (data not shown). Then, we monitored the glutamine uptake from the culture medium using HPLC analysis. Although glutamine and histidine shared similar retention time during HPLC analysis, we did not observe any significant change in glutamine/ histidine transport (data not shown). We quantified the intracellular glutamine levels by NMR and observed its considerable increase after QB treatment, suggesting the inhibition of glutaminolysis and/or glutamine-dependent reactions (e.g. hexosamine and de novo purine synthesis) after QB treatment (Figure 4C). Next, we probed the effect of QB on glutamate transport and intracellular levels using NMR or HPLC, respectively. Using NMR, we observed a decrease in the intracellular glutamate levels, but on using HPLC, we observed an increase in the extracellular glutamate levels, suggesting an increased transport of glutamate out of the cell during QB treatment (Figure 4D and Figure 4E). Moreover, on using LFQ, we observed a significant decrease in enzymes involved in glutamine metabolism, such as glutaminase (GLS), GDH and alpha-ketoglutarate dehydrogenase (OGDH; Figure 4F, 4G and 4H). Unfortunately, we were unable to detect the levels of other metabolites of glutamine metabolism (from AKG to succinate), but we confirmed the increased levels of succinate (Figure 1C) after QB treatment as a result of complex II inhibition (confirmed also by oxygraph measurements). Finally, we determined the levels of aspartate after QB treatment and observed a slight decrease in aspartate levels in leukemic cells (Figure 4I). We also confirmed blockade of aspartate uptake from cell culture medium by Jurkat leukemic cells (data not

\section{A}
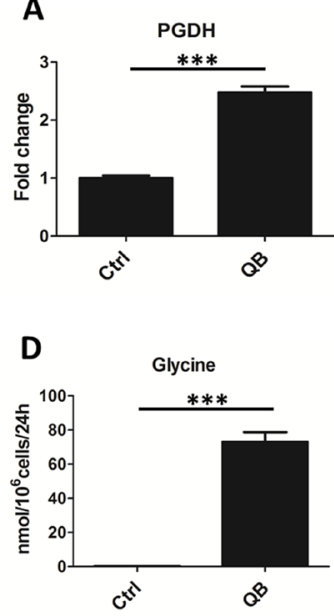

H

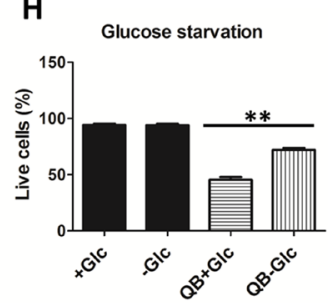

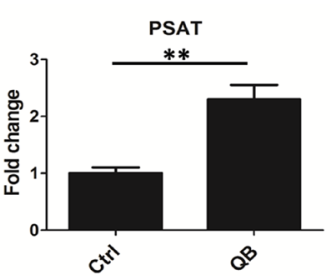

E
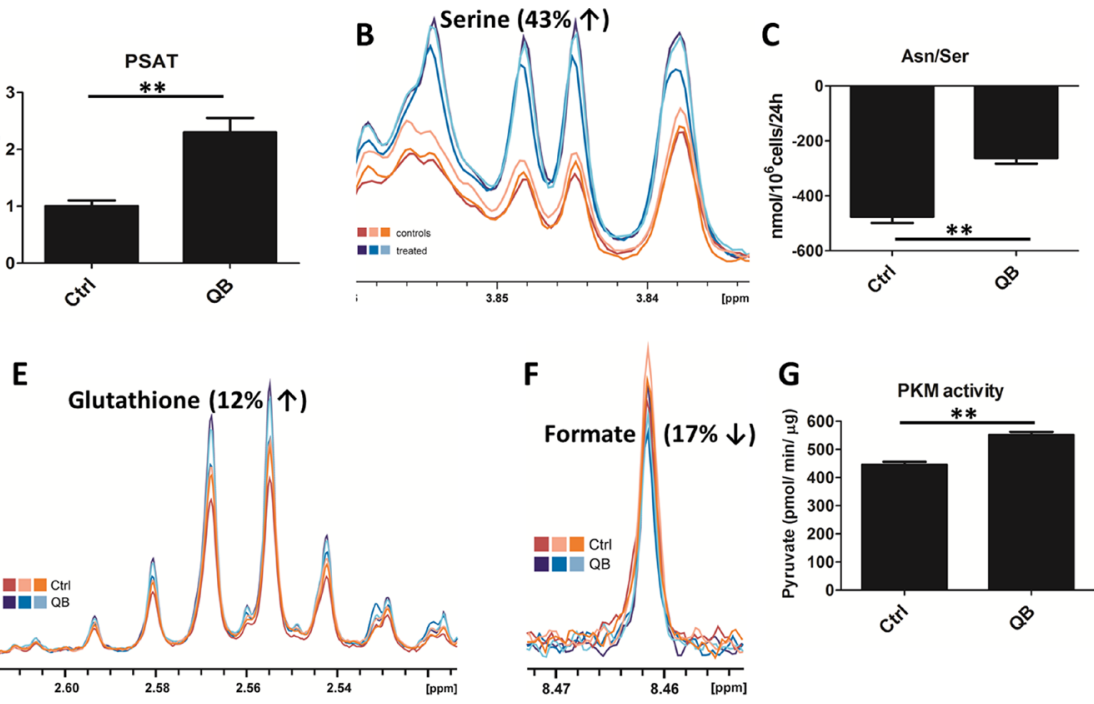

Figure 3: Effect of Quambalarine B (QB) on serine-glycine synthesis pathway. (A) Relative levels of individual enzymes of serine synthesis pathway in control (ctrl) and QB-treated cells (QB) determined by LFQ analysis. (B) Intracellular levels of serine in control (orange lines) and QB-treated (blue lines) cells determined by NMR analysis. (C) Changes in Asn/Ser levels in culture medium after $24 \mathrm{~h}$ of control (ctrl) and QB-treated cells (QB) determined by HPLC analysis. (D) Changes in glycine levels in culture medium after $24 \mathrm{~h}$ of control (Ctrl) and QB-treated cells (QB) determined by HPLC analysis. (E) Intracellular levels of glutathione in control (orange lines) and QB-treated (blue lines) cells determined by NMR analysis. (F) Intracellular levels of formate in control (orange lines) and QBtreated (blue lines) cells determined by NMR analysis. (G) Pyruvate kinase M (PKM) activity in control (Ctrl) and QB-treated cells (QB) determined by enzymatic assay. (H) Percentage of dead cells in normal cultures and cultures under glucose starvation after QB treatment were determined by FACS analysis. Data are shown as means from three independent experiments \pm SEM. ${ }^{*}$, significant differences with $P<0.05$. ${ }^{* *}$, significant differences with $P<0.01$. $^{* * *}$, significant differences with $P<0.001$. The images shown are representative of three independent experiments. In all experiments cells were treated with $2 \mu \mathrm{mol} / \mathrm{L}$ of QB 
shown). Inhibition of aspartate synthesis represented intriguing mechanism responsible for inhibition of cellular proliferation triggered by QB treatment.

\section{QB strongly deregulates levels of amino acids in leukemic cells}

Imbalance in AA metabolism represents an important hallmark of cancer cells with potential applications in cancer therapy $[36,37]$. To explore the effect of QB on AA milieu in leukemic cells, we determined the uptake of individual AA using HPLC and quantified the intracellular levels of AA after $24 \mathrm{~h}$ of QB treatment using NMR analysis. We detected an increase in the intracellular levels of glycine, isoleucine, leucine, valine, asparagine, threonine, alanine, serine and glutamine and a decrease in the intracellular levels of glutamate and aspartate (Figures $1 \mathrm{E}, 3 \mathrm{~B}, 4 \mathrm{C}, 4 \mathrm{D}, 4 \mathrm{I}$, and 5A).

We observed no changes in isoleucine, leucine and valine uptake (essential AA), but we confirmed a significant decrease in threonine and arginine uptake. These results suggest the mechanism responsible for AA accumulation in Jurkat cells other than upregulated transport from the culture medium (Figure 5B). Suspect mechanisms can involve inhibition of protein translation and/or induction of autophagy by QB in leukemic cells. We identified the unexpected effect of $\mathrm{QB}$ on the uptake of alanine using HPLC. Control cells imported a significant amount of
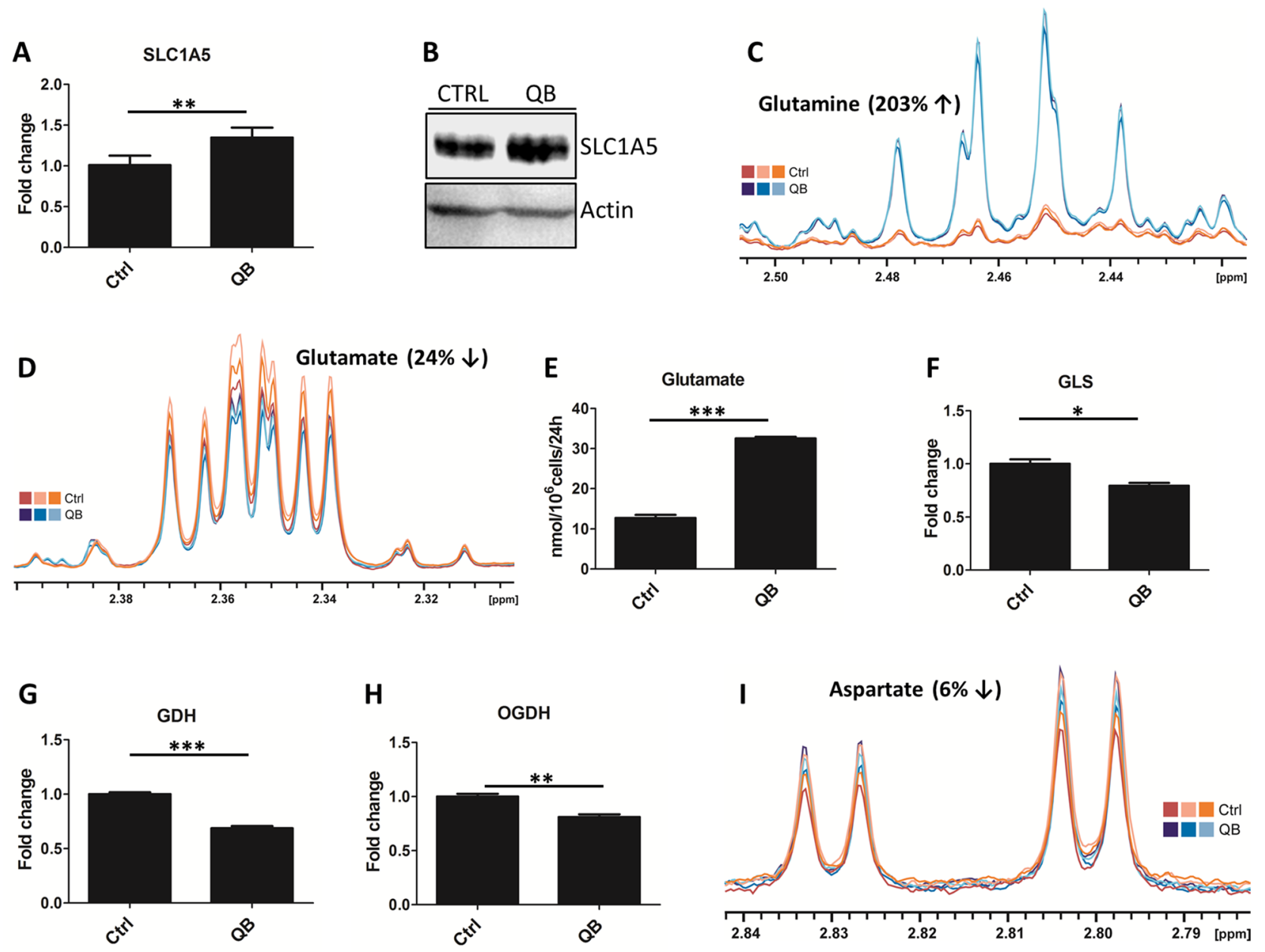

Figure 4: Quambalarine B (QB) deregulates glutamine metabolism in leukemic cells. (A) Relative levels of glutamine transporter SLC1A5 in control (ctrl) and QB-treated (QB) cells determined by LFQ analysis. (B) Levels of SLC1A5 transporter in control (CTRL) and QB-treated cells visualized by immunoblot. (C) Intracellular levels of glutamine in control (orange lines) and QB-treated (blue lines) cells determined by NMR analysis. (D) Intracellular levels of glutamate in control (orange lines) and QB-treated (blue lines) cells determined by NMR analysis. (E) Changes in glutamate levels in the culture medium after $24 \mathrm{~h}$ of control (ctrl) and QB-treated (QB) cells determined by HPLC analysis. (F, G, H) Relative levels of glutamine metabolism enzymes: glutaminase (GLS), glutamate dehydrogenase (GDH), and alpha-ketoglutarate dehydrogenase (OGDH) in control (ctrl) and QB-treated (QB) cells determined by LFQ analysis. (I) Intracellular levels of aspartate in control (orange lines) and QB-treated (blue lines) cells determined by NMR analysis. Data are shown as means from three independent experiments \pm SEM. *, significant differences with $P \leq 0.05^{* *}$, significant differences with $P \leq 0.01^{* * *}$, and significant differences with $P \leq 0.001$. The images shown are representative of three independent experiments. In all experiments cells were treated with $2 \mu \mathrm{mol} / \mathrm{L}$ of QB. 
alanine from the culture medium. In contrary, the QBtreated cells exported a significant amount of alanine into medium (Figure 1F). Extracellular alanine could originate from glycolysis as a result of the pyruvate transamination, autophagy or inhibition of protein translation. Serine represented another nonessential AA, which was accumulated in the leukemic cells during QB treatment. Although serine retention time matched the asparagine characteristics during HPLC analysis, we observed a significant decrease in asparagine/serine uptake during QB treatment (Figure 3C). Based on these observations, we emphasized on increased synthesis of serine as a result of rewired glycolytic flux triggered by QB in Jurkat cells, autophagy or inhibition of protein synthesis. Glutamine represented the most accumulated AA in leukemic cells after QB treatment (Figure 4C). Although glutamine retention time matched to that of histidine retention time during HPLC analysis, no effect of QB treatment on glutamine/ histidine transport was observed. Accumulation of glutamine seemed to originate from mitochondrial complex II blockade, nucleotides and hexosamine biosynthesis inhibition and/or autophagy together with protein translation
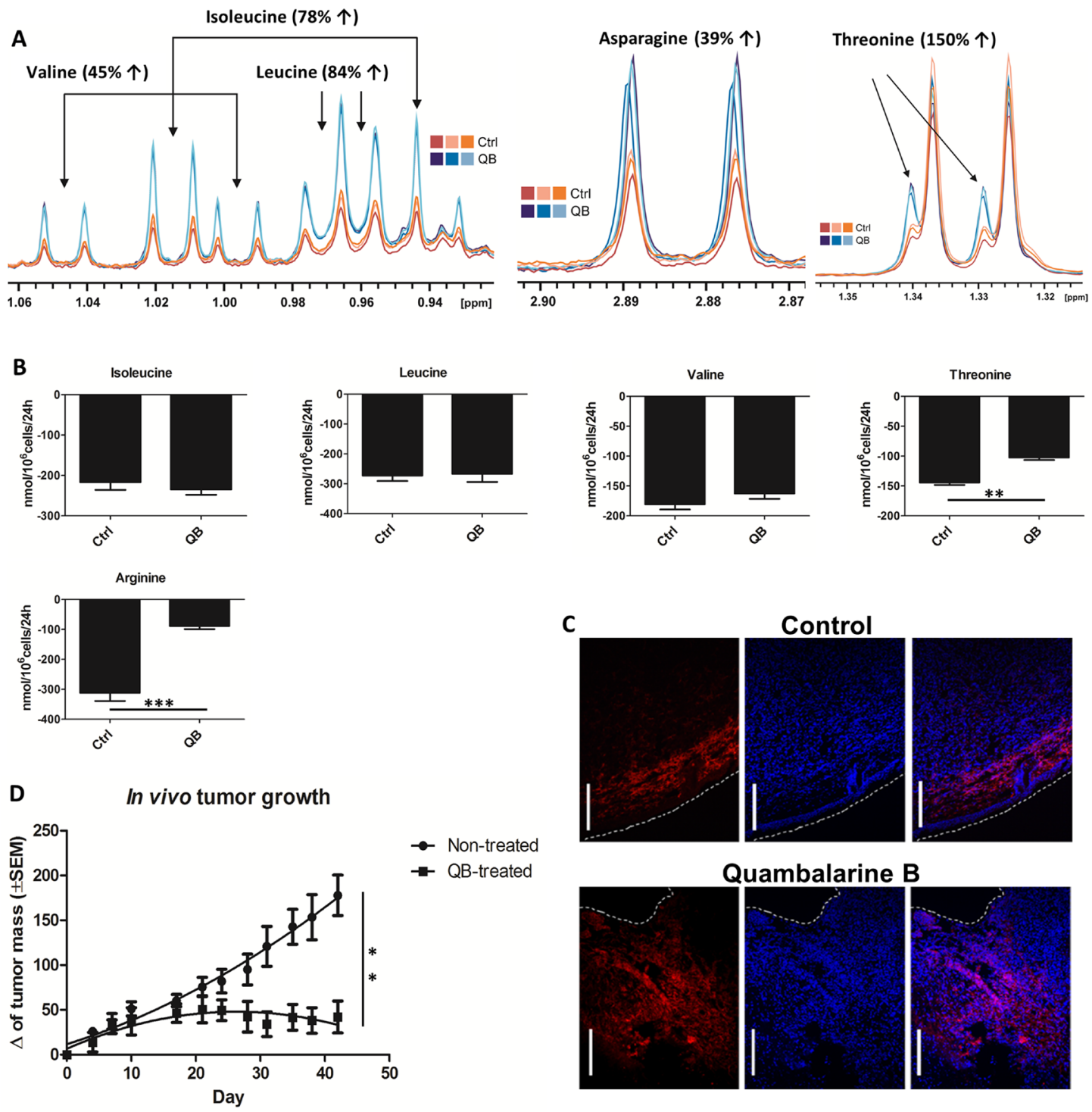

Figure 5: Quambalarine B (QB) increases levels of several AA in leukemic cells and inhibits tumor growth in vivo. (A) Intracellular levels of valine, leucine, isoleucine, asparagine and threonine in control (orange lines) and QB-treated (blue lines) cells determined by NMR analysis. (B) Changes in individual AA levels in the culture medium of control (ctrl) and QB-treated (QB) cells after $24 \mathrm{~h}$ incubation determined by HPLC analysis. (C) Distribution of macrophages (F4/80+) in solid tumors derived from human breast cancer cell line MDA-MB-231 developed and treated with QB in the immunocompromised mouse model (blue - cell nuclei visualized by DAPI, red - mouse macrophages (F4/80+), dashed white line - tumor edge, white bar corresponds to $100 \mu \mathrm{m})$. (D) Effect of QB on tumor growth in vivo. Results from in vivo experiment represent mean \pm SEM of value from one experiment (5 mice per group). All data are shown as means from three independent experiments \pm SEM. ${ }^{*}$, significant differences with $P \leq 0.05$. $^{* *}$, significant differences with $P \leq 0.01{ }^{* * *}$, and significant differences with $P \leq 0.001$. 
stop. Since glutamine represents the most consumed AA by leukemic cells in a medium, we did not anticipate the involvement of glutamine synthesis in this process. Asparagine represents the second favorite AA, which has been eaten by leukemic cells from the culture medium, and the HPLC technique showed decreased asparagine/serine uptake by Jurkat cells during QB treatment (Figure 3C). Hence, increased intracellular levels of asparagine can be a result of autophagy and/or inhibition of protein translation as well. Glutamate represented AA, which can be generated in cells as a result of glutamine deamination catalyzed by GLS or glutamine-dependent enzymes. Surprisingly, leukemic cells did not uptake glutamate from the culture medium and rather export excess of glutamate into the medium. We observed increased production of glutamate by Jurkat cells into the medium after QB treatment, which can be a result of autophagy activation, protein translation inhibition or can reflect inhibition of TCA cycle that is fed mainly through glutaminolysis (Figure 4E). A similar effect was observed for glycine during QB treatment. Control cells did not transport any glycine from the culture medium, but QB-treated cells produced a significant amount of glycine into the medium. Finally, aspartate represented the only AA, which was generally decreased in Jurkat cells after QB treatment, and we detected neither uptake nor export of aspartate by leukemic cells. Since the synthesis of aspartate by leukemic cells seemed to be blocked by QB, we presumed autophagy and/or inhibition of protein synthesis as the mechanisms buffering aspartate levels under QB pressure.

\section{QB maintains its anti-tumor activity in in vivo mouse xenograft model}

For the in vivo test of the anti-tumor activity of $\mathrm{QB}$, the cell line MDA-MB-231 (human breast carcinoma) was used. Cells were introduced subcutaneously, and the size of the developing tumors was measured. The result clearly showed inhibition of the tumor growth on a statistically sufficient set of animals. At the end of the experiment, tumors were removed and subjected to immunohistological study comparing the control vs. treated tumors. A clear distinction was observed in the pattern and quantity of tumor infiltrating macrophages (F4/80+ cells). In control tumors, the macrophages were concentrated in a sharp line close to the tumor capsule. In tumors removed from the mouse treated with QB, macrophages infiltration was massive and reached much deeper into the tumor. Clear distinction was also found in the histological composition of the cancer edge. Control tumors were enveloped by E-cadherin highly positive stratified mouse epithelia, whereas the surface of tumors from treated mice was "eroded" with much less epithelial cover (data not shown). Data from in vivo mouse xenograft model clearly supported the notion that QB maintained its anti-tumor activity in in vivo conditions (Figure 5C and 5D).

\section{DISCUSSION}

We present here the proof of principle that metabolo-proteomic approach could be useful for deciphering molecular mechanisms underlying novel drugs action. Molecular simulations pointed out mitochondrial respiratory complexes I and II as potential targets of QB in Jurkat cells together with ubiquinonecompetitive properties of QB. Mitochondrial respiratory complexes were recently showed as intriguing targets for cancer and diabetes therapy [38-40]. As the activity of these complexes is tightly linked to other metabolic pathways, we tracked alterations in these pathways during treatment of leukemic cells with $\mathrm{IC}_{50}$ concentration of QB as determined by MTS assay. The treatment showed the inhibitory effect on cellular proliferation, did not induce cell death and led to the rearrangement of metabolism of Jurkat cells [24]. Inhibition of ETC by QB led to increased levels of intracellular AMP, which inhibited anabolic pathways crucial for leukemic cells proliferation. AMP is a well-known stimulator of PFK activity, which preferentially feeds downstream parts of glycolysis to support anaplerotic substrates for mitochondrial metabolism. Enhanced PFK activity results in depletion of upstream glycolytic intermediates crucial for pentose and hexosamine biosynthetic pathways. As a potential result of high AMP levels, we confirmed a decrease in O-glycosylation of proteins, which was represented as an important regulator of stability of several oncogenic factors such as C-MYC. Consistent with this hypothesis, C-MYC levels were significantly downregulated during QB treatment [24]. Hence, inhibition of hexosamine pathway and protein O-glycosylation provided a new possibility to regulate leukemia cells proliferation [41]. In agreement with these findings, we detected increased levels of glycolysis-derived intermediates feeding mitochondrial metabolism represented by pyruvate, succinate, and serine. Inhibition of mitochondrial complexes I and II by QB slowed down the utilization of electron donors by ETC followed by accumulation, conversion, and export of such metabolites out of the cell. These reactions involved conversions of serine to glycine and pyruvate to alanine together with the inhibition of pyruvate to lactate conversion. Glycine can be metabolized to toxic products, amino acetate and methylglyoxal, a reactive aldehyde, which covalently modifies AA residues in proteins [31]. Export of glycine out of cell seemed to represent an important protective mechanism in leukemia cells during ETC blockade. This fact highlighted glycine export machinery as a novel target for leukemia therapy. On the other hand, alanine acted as a potent inhibitor of PKM activity and pyruvate synthesis resulting in accumulation of upstream glycolytic intermediates [33]. Hence, metabolism and transport of alanine represented other potential therapeutic target. Next, serine acted as an allosteric activator of PKM activity, which was increased 
during QB treatment [34]. Inhibition of serine to glycine conversion can increase lactate levels and toxicity in cancer cells due to PKM activity stimulation as well as inhibit folate-driven ETC activity and purine biosynthesis. Contrary to our findings, recent reports claimed the inhibitory effect of naphthoquinonic compound shikonin on PKM activity in cancer cells [42]. Since shikonin possesses unsubstituted C2 position in its structure, we presume covalent binding of shikonin to regulatory cysteines in PKM molecule as a potential mechanism responsible for shikonin-driven inhibition of PKM [43]. Consistent with this assumption, we observed activation of ERK kinase by shikonin in Jurkat cells as a potential result of regulatory phosphatases inhibition (Supplementary Figure 2) [22].

We have also detected increased levels and export of several AA, except aspartate, in Jurkat cells during QB treatment. Increased levels of AA can originate from de novo synthesis as well as autophagy or inhibition of protein translation. Aspartate represents one of the key building blocks for nucleotide and protein synthesis. Jurkat cells showed extremely low uptake of aspartate from culture medium and were strongly dependent on aspartate synthesis from oxaloacetate [9]. We suggest that when oxaloacetate synthesis became inhibited by QB through complex II blockade, the levels of aspartate were transiently supported by autophagy followed by proliferation arrest. Increased levels of intracellular glutamine and extracellular glutamate also suggest inhibition of mitochondrial metabolism during QB treatment. These results demonstrated inhibition of aspartate synthesis as a promising therapeutic target for leukemia therapy. Moreover, inhibition of complex I activity together with the effect of QB on glycolytic flux rendered QB as a potential anti-diabetic drug. Several well-known anti-diabetic drugs act as inhibitors of ETC and decrease glucose levels in patient's serum [44]. Since QB anti-cancer activity was confirmed also during in vivo studies, the practical therapeutic potential of QB will be extensively tested in the future.

\section{MATERIALS AND METHODS}

\section{Cell line, culture conditions and reagents}

The T-cell lymphoma-derived Jurkat cell line, clone E6.1, was obtained from the ATCC collection (ATCC, Manassas, VA, USA) and cultured at $37^{\circ} \mathrm{C}$ and $5 \% \mathrm{CO}_{2}$ in the RPMI1640 medium supplemented with L-glutamine (Lonza Group, Ltd., Basel, Switzerland), 10\% fetal bovine serum (FBS) (Gibco, USA), $100 \mathrm{U} / \mathrm{mL}$ penicillin, and $100 \mu \mathrm{g} / \mathrm{mL}$ streptomycin (PAA Laboratories, Austria). Cells were treated with $2 \mu \mathrm{mol} / \mathrm{L}$ concentration of QB. Unless otherwise stated, cells were seeded at a density of $2.5 \times 10^{5}$ cells $/ \mathrm{mL}$ in $15 \mathrm{~mL}$ of the RPMI1640 medium, cultured overnight in $75 \mathrm{~cm}^{2}$ cell culture flasks under standard cultivation conditions, treated with QB for 24 $\mathrm{h}$ and together with control cells immediately counted, harvested, washed with PBS and stored in deep freezer for subsequent analysis. For all experiments, QB stock solution was used $\left(25 \mathrm{mmol} / \mathrm{L}\right.$ in DMSO, kept in $\left.-20^{\circ} \mathrm{C}\right)$.

\section{RNA isolation, reverse transcription and RT- qPCR analysis}

Cells were pelleted $(400 \times \mathrm{g}, 4 \mathrm{~min})$, washed with PBS buffer and lysed in a cell lysis buffer (Aurum RNA Isolation Kit, Bio-Rad, USA). Cell lysate was processed according to the manufacturer's protocol, including an in-column DNase treatment. The purified RNA was quantified and tested for the presence of contaminants with a NanoDrop spectrophotometer (Thermo Fisher Scientific, USA). Reverse transcription of $700 \mathrm{ng}$ of each purified RNA sample was performed using the M-MulV Taq RTPCR Kit (New England Biolabs, USA). For individual RTqPCR reaction mixtures, $2 \mu \mathrm{L}$ of cDNA from the reverse transcription protocol were used. Individual reactions were run in triplicate using a CFX96 Real-Time PCR System (Bio-Rad, USA) in final volumes of $25 \mu \mathrm{L}$. Specific primers and the SsoFast ${ }^{\mathrm{TM}}$ EvaGreen Supermix (Bio-Rad, USA) were used for amplification and fluorescent detection of PCR products, respectively. The relative quantities of cDNA from treated and control cells were calculated by the Livak and Schmittgen $2^{-\Delta \Delta C t}$ method [45]. P0 was used as a reference gene. Following primers were used for specific gene amplification: GLUT1-Forward TCGT CGTC GGCA TCCT CATC, GLUT1-Reverse CGGTTGATGAGCAGGAAGCG; P0-Forward TCGA CAAT GGCA GCAT CTAC, P0-Reverse ATCC GTCT CCAC AGAC AAGG.

\section{SDS electrophoresis and immunoblotting}

For whole cell lysate analyses, cells were washed with PBS supplemented with a Phosphatase Inhibitor Cocktail (Active Motif, Belgium), lysed using the RIPA buffer ( $1 \% \mathrm{NP}-40,150 \mathrm{mmol} / \mathrm{L} \mathrm{NaCl}, 0.5 \% \mathrm{NaDOC}, 0.1 \%$ SDS and $50 \mathrm{mmol} / \mathrm{L}$ Tris-HCl $\mathrm{pH}$ 8) supplemented with Complete Protease Inhibitor Cocktail Tablets (F. HoffmannLa Roche Ltd., Switzerland), and incubated for $30 \mathrm{~min}$ on ice. The cell lysate was cleared via centrifugation $(14000 \times$ $\mathrm{g}, 10 \mathrm{~min}, 4^{\circ} \mathrm{C}$ ). Bicinchoninic acid (BCA) assay was used to determine total protein concentration in the cell lysates (BCA Protein Assay Kit, Thermo Fisher Scientific, USA). Nuclear and cytosolic fractions were isolated from Jurkat cells using Nuclear Extract Kits (Active Motif, Belgium). SDS PAGE gels were loaded with $40 \mu \mathrm{g}$ of protein per lane from individual samples. A Protean III apparatus (Bio-Rad, USA) with a constant voltage of $100 \mathrm{~V}$ was used to run the SDS PAGE protein samples. Separated proteins were blotted onto a nitrocellulose membrane (GE Healthcare, USA) using a Trans-Blot ${ }^{\mathrm{TM}}$ SD Semi-Dry apparatus (BioRad, USA). Protein blots were blocked for $1 \mathrm{~h}$ in TBS 
supplemented with 5\% milk (Bio-Rad, USA) and 0.05\% Tween-20 (Sigma-Aldrich). Membranes were then washed with TBS containing $0.05 \%$ Tween-20 and incubated with the respective primary and secondary antibodies, according to manufacturer's protocols. The following antibodies were used for immunostaining: anti-GLUT1 and anti-SLC1A5 rabbit primary antibodies (Abcam, UK). Secondary antibody against rabbit IgG were purchased from Santa Cruz Biotechnology.

\section{Effect of glucose deprivation on response of Jurkat cell line to QB}

Jurkat cells were seeded in 24-well plate at a density $2 \times 10^{5}$ cells $/ \mathrm{mL}$ and cultured in final volume of $1 \mathrm{~mL}$ in complete RPMI1640 medium or medium lacking glucose. After $24 \mathrm{~h}$ incubation with $\mathrm{EC}_{50}$ of $\mathrm{QB}$, fractions of live, apoptotic and dead cells were determined using Hoechst 33258 staining by flow cytometry measurement (FACS LSRII instrument, BD Biosciences, San Jose, CA, USA). Cells cultured in RPMI1640 with DMSO only were used as a control. Data analysis was performed using the FlowLogic 600.A software (Inivai Technologies, Australia).

\section{Metabolite extraction}

The extraction was performed using $10^{7}$ cells per sample according to Gottschalk et al. protocol [46]. Cells were incubated according to standard protocol, counted, pelleted into a $1.5 \mathrm{~mL}$ vial and washed with PBS. Then 600 $\mu \mathrm{L}$ of a $2: 1(\mathrm{v} / \mathrm{v})$ ratio of ice-cold methanol/chloroform was added, the pellets were re-suspended using a vortex mixer and incubated for $10 \mathrm{~min}$ on a rotator. Then $600 \mu \mathrm{L}$ of ice-cold 1:1 (v/v) chloroform/water was added, mixed using a vortex and sonicated in the sonicating bath for 10 min. Samples were then centrifuged at $13000 \mathrm{rpm}$ for $5 \mathrm{~min}$. The top layer was removed to a new vial taking care not to disturb the pelleted debris. The solvent was evaporated using a centrifugal concentrator and samples were frozen $\left(-80^{\circ} \mathrm{C}\right)$ for later analysis.

\section{NMR metabolomics}

The dried hydrophilic cell extracts were re-dissolved in a mixture of $450 \mu \mathrm{L} \mathrm{D}_{2} \mathrm{O}$ and $50 \mu \mathrm{L}$ deuterated potassium buffer $\left(1.5 \mathrm{~mol} / \mathrm{L} \mathrm{KH} 2 \mathrm{PO} 4\right.$ in $\mathrm{D}_{2} \mathrm{O}$ containing $2 \mathrm{mmol} / \mathrm{L}$ $\mathrm{NaN} 3$ and $0.1 \%$ trimethylsilyl propionic acid (TSP) as an internal standard; $\mathrm{pH}=7.4$ ) and transferred to a 5-mm NMR tube. The NMR spectra were recorded on a $600 \mathrm{MHz}$ Bruker Avance III spectrometer (Bruker BioSpin, Rheinstetten, Germany) equipped with a 5-mm TCI cryogenic probe head.

All NMR experiments were performed at $298 \mathrm{~K}$. Standard 1H NMR spectra were acquired using nuclear Overhauser enhancement spectroscopy (1D-NOESY) pulse sequence with following acquisition parameters: number of scans (NS) $=512,64 \mathrm{k}$ of data points (TD), spectral width (SW) of $20 \mathrm{ppm}$, relaxation delay (D1) of 4 $\mathrm{s}$, a mixing time (D8) of $10 \mathrm{~ms}$. The resonances of water were suppressed by presaturation during relaxation delay and mixing time. J-resolved experiment with presaturation $(\mathrm{NS}=16, \mathrm{SW}=16 \mathrm{ppm}, \mathrm{TD}=16 \mathrm{k}$, number of increments $=40, \mathrm{SW}=78.125 \mathrm{~Hz}$ in the indirect dimension, and relaxation delay $=2 \mathrm{~s}$ ) was performed to facilitate the identification of metabolites. Additionally, 2D correlation spectroscopy (COSY) and heteronuclear single-quantum correlation experiments (HSQC) were measured for selected samples using standard manufacturer's software Topspin 3.2 (Bruker BioSpin, Rheinstetten, Germany).

The acquired free induction decays (FIDs) were multiplied by an exponential window function (LB $=0.3 \mathrm{~Hz}$ ). The spectra were automatically phased, baseline corrected and referenced to TSP $(0.0 \mathrm{ppm})$. The processed 1D-NOESY spectra $(0.10-10.0 \mathrm{ppm})$ were uniformly binned and normalized to the total spectral area in Amix 3.9.14 software (Bruker Biospin, Rheinstetten, Germany) using the 0.01 binning step. The intensity of the respective signal, i.e. metabolite, was then determined as the sum of the corresponding $0.01-\mathrm{ppm}$ bins to cover the whole signal width. The changes of some metabolites (glycine, threonine) were due to overlap with signals of other metabolites determined using Chenomx NMR Suite 7.6 (Chenomx Inc., Edmonton, AB, Canada). Regions corresponding to water (4.70-4.90 ppm) were removed prior to binning process. The peak assignment was performed also using Chenomx NMR Suite 7.6, and the HMDB database and published assignments [47]. The metabolite identification was supported by J-resolved and COSY experiments and confirmed using 2D NMR experiments.

\section{HPLC analysis of amino acids uptake from culture medium}

Cells were seeded in six-well plates at concentration $10^{6}$ cells $/ \mathrm{mL}$ and treated with QB. Cell were then counted using Bürker counting chamber, pelleted by centrifugation (400 x g, $4 \mathrm{~min}$ ), medium was removed and centrifuged $(12000 \mathrm{x} \mathrm{g}, 10 \mathrm{~min})$ to remove cell debris. Medium incubated in six-well plates without cells was used as a control. High performance liquid chromatography analysis of amino acids was performed using Waters AccQ-Tag Chemistry Package (WAT052875) on two pump Beckman Coulter Gold chromatograph with Merck-Hitachi F-1080 fluorescence detector (ex.250nm, em. $395 \mathrm{~nm}$ ). Data were collected and evaluated with DataApex CSW32 chromatography software.

\section{Determination of glucose uptake from medium}

Cells were seeded in six-well plates at concentration 5 $\times 10^{5}$ cells $/ \mathrm{mL}$ and treated with QB or corresponding DMSO. After incubation, cells were counted using Bürker counting 
chamber, pelleted (400 g, 4 min, RT) and supernatant was centrifuged again (14000 g, $10 \mathrm{~min}, \mathrm{RT})$ to eliminate cell debris. Final supernatant and control medium were diluted 50 times by adding $\mathrm{dH}_{2} \mathrm{O}$, glucose concentration was determined using Glucose (GO) Assay Kit (Sigma-Aldrich, USA) according to the manufacturer protocol.

\section{Determination of cellular fructose-6-phosphate levels}

To determine the fructose 6-phosphate (F6P) concentration, the PicoProbeTM Fructose 6-Phosphate Fluorometric Assay Kit (BioVision, USA) was used. Cells were cultivated in $50 \mathrm{ml}$ of medium in $150 \mathrm{~cm}^{2}$ flasks at concentration $20 \times 10^{6}$ and treated with QB. To deproteinize the samples, cells were lyzed in $200 \mu \mathrm{l}$ of $\mathrm{dH}_{2} \mathrm{O}$ and then centrifuged (14 $000 \mathrm{~g}, 10 \mathrm{~min}, \mathrm{RT}$ ). Supernatants were transferred to the centrifugal filter units with molecular weight cut-off of $10 \mathrm{kDa}$ (Merck Millipore, Germany) and F6P was detected in the acquired filtrates according to the manufacturer protocol.

\section{Determination of lactate production}

Lactate production was determined using Lactate Assay Kit (Sigma-Aldrich, USA). Cells were seeded at concentration $2.5 \times 10^{5}$ cells $/ \mathrm{mL}$ and treated with $\mathrm{QB}$ or DMSO (control samples) for $24 \mathrm{~h}$. After treatment cells were transferred to six-well plate at concentration of $10^{6}$ cells/mL and after $8 \mathrm{~h}$ centrifuged (400 g, $4 \mathrm{~min}$ and 12 $000 \mathrm{~g}, 10 \mathrm{~min}$ RT). Supernatant was analyzed for the lactate concentration according to manufacturer protocol.

\section{Determination of PKM kinetics}

PKM kinetics was determined using Pyruvate Kinase Activity Kit (BioVision, USA). Cells were seeded at concentration $2.5 \times 10^{5}$ cells $/ \mathrm{mL}$ and directly treated with QB or DMSO. Cells were then lysed using Lysis buffer (BioVision, USA) and protein concentration was determined using BCA assay. One $\mu \mathrm{g}$ of extracted proteins from QB-treated and control cells was used for PKM activity determination in fluorescence mode.

\section{Determination of pyruvate cellular levels}

Cells were cultivated and treated according to standard protocol. Pyruvate concentrations were determined in equal amounts of cells using Pyruvate Kinase Activity Kit (BioVision, USA) according to the manufacturer protocol.

\section{Label-free quantification analysis (LFQ)}

To determine changes in Jurkat cell proteome caused by QB treatment, label-free quantification analysis was performed. We rely on sodium deoxycholate (SDC) and phase-transfer surfactant (PTS) method [48]. Cell pellets $\left(1-2 \times 10^{6}\right.$ cells $)$ were lyzed in $100 \mathrm{mmol} / \mathrm{L}$ triethylammonium bicarbonate (TEAB) containing $2 \%$ SDC (50 $\mu$ l of buffer/ 1 million of cells). To eliminate DNA, cell lyzates were sonicated (10 min or until clear) or treated with benzonase $(50 \mathrm{U} / \mathrm{mL}, 30 \mathrm{~min}$ on ice). Thereafter, the samples were boiled for $5 \mathrm{~min}$ and centrifuged (14 $000 \mathrm{~g}$, 5 min, RT). Bicinchoninic assay (Thermo Fisher Scientific, USA) was used to determine protein concentration in the supernatants, the amount of $20 \mu \mathrm{g}$ of proteins was sufficient for a mass spectrometric measurement. Following cysteines reduction by $5 \mathrm{mmol} / \mathrm{L}$ triscarboxyethyl phosphine (TCEP; $30 \mathrm{~min}, 60^{\circ} \mathrm{C}$ ), cysteines were alkylated using methyl methanethiosulfonate (MMTS) of the final concentration of $10 \mathrm{mmol} / \mathrm{L}$ (10 min, RT). The next step involved enzymatic digestion by trypsin in the ratio 1:20 (w/w) trypsin:proteins (overnight, $37^{\circ} \mathrm{C}$ ), then the reaction was stopped by adding trifluoracetic acid (TFA) to the final concentration of $0.5 \%$. Finally, SDC was removed from samples using ethylacetate (PTS method) and peptides were desalted (peptide MicroTrap column, C18 phase, Michrom Bioresources, USA).

\section{nanoLC-MS ${ }^{2}$ analysis}

Nano reversed phase columns (EASY-Spray column, $50 \mathrm{~cm} \times 75 \mu \mathrm{m}$ ID, PepMap C18, $2 \mu \mathrm{m}$ particles, $100 \AA$ pore size) were used for LC/MS analysis. Mobile phase buffer A was composed of water, $2 \%$ acetonitrile and $0.1 \%$ formic acid. Mobile phase B was composed of $80 \%$ acetonitrile and $0.1 \%$ formic acid. Samples were loaded onto the trap column (Acclaim PepMap300, C18, $5 \mu \mathrm{m}$, $300 \AA$ Wide Pore, $300 \mu \mathrm{m} \times 5 \mathrm{~mm}, 5$ Cartridges) for $4 \mathrm{~min}$ at $15 \mu \mathrm{l} / \mathrm{min}$. Loading buffer was composed of water, $2 \%$ acetonitrile and $0.1 \%$ trifluoroacetic acid. Peptides were eluted with mobile phase B gradient ( $2 \%$ to $40 \%$ ) in 120 min. Eluted peptide cations were converted to gas-phase ions by electrospray ionization and analyzed on a Thermo Orbitrap Fusion (Q-OT- qIT, Thermo Scientific, USA). Survey scans of peptide precursors from 400 to $1600 \mathrm{~m} / \mathrm{z}$ were performed at $120 \mathrm{~K}$ resolution (at $200 \mathrm{~m} / \mathrm{z}$ ) with a $5 \times 10^{5}$ ion count target. Tandem MS was performed by isolation at $1.5 \mathrm{Th}$ with the quadrupole, HCD fragmentation with normalized collision energy of 30 , and rapid scan MS analysis in the ion trap. The $\mathrm{MS}^{2}$ ion count target was set to 104 and the max injection time was $35 \mathrm{~ms}$. Only those precursors with charge state 2-6 were sampled for $\mathrm{MS}^{2}$. The dynamic exclusion duration was set to $45 \mathrm{~s}$ with a $10 \mathrm{ppm}$ tolerance around the selected precursor and its isotopes. Monoisotopic precursor selection was turned on. The instrument was run in top speed mode with 2 s cycles.

\section{LFQ data analysis}

MaxQuant 1.5.3.8 peak picking software and Andromeda search engine were used in label-free experiments. Followed parameters were set during 
measurement: enzyme specificity considered- Trypsin/P, number of missed cleavages permitted - 2, fixed modification(s) - S-Methyl-L-cysteine sulphoxide (C), variable modification - Oxidation (M), Acetyl (Protein $\mathrm{N}$-term), mass tolerance for precursor ions $-4.5 \mathrm{ppm}$ and mass tolerance for fragment ions - 0.5 Da. False discovery rate (FDR) was set as follows: peptide spectrum match FDR $-1 \%$, Protein FDR $-1 \%$ and min. score for modified peptides -40 . Human protein database downloaded from uniprot.org on 9/15/2015 with 147933 entries was used for individual proteins annotation. The "match between runs" feature of MaxQuant was used to transfer identifications to other LC-MS/MS runs based on their masses and retention time (maximum deviation $0.7 \mathrm{~min}$ ). This setup was also used in quantification experiments. Quantifications were performed with the label-free algorithms described recently [49]. All MS experiments were done in biological triplicates and measured in technical duplicates.

\section{Mitochondria isolation procedure}

Liver was dissected out from sacrificed rat (Wistar, Animal facility of Institute of physiology CAS) and put in ice-cold isolation medium $(0.25 \mathrm{~mol} / \mathrm{L}$ saccharose, 20 mmol/L TRIS, 1 mmol/L EDTA, pH 7.4). Approximately $5 \mathrm{~g}$ of the tissue was taken for isolation, minced with scissors in ice-cold isolation medium, washed and then re-suspended with ice-cold isolation medium and homogenized with Potter-Elvehjem homogenizer (glassteflon) by six strokes at $800 \mathrm{rpm}$ using a digital overhead stirrer (VELP Scientifica). Homogenate was transferred into two $50 \mathrm{ml}$ Falcon tubes and centrifuged at $800 \mathrm{~g}, 10$ $\min , 4^{\circ} \mathrm{C}$, supernatant was centrifuged $(10000 \mathrm{~g}, 10 \mathrm{~min}$, $4^{\circ} \mathrm{C}$ ), washed with ice-cold isolation medium, centrifuged (10 $000 \mathrm{~g}, 10 \mathrm{~min}, 4^{\circ} \mathrm{C}$ ), washed with ice-cold isolation medium, transferred into 2 tubes and centrifuged (10 $000 \mathrm{~g}, 10 \mathrm{~min}, 4^{\circ} \mathrm{C}$ ). Finally, purified mitochondria were re-suspended in ice-cold isolation medium, kept on ice and immediately used for high-resolution respirometry measurement by OROBOROS Oxygraph-2k instrument (Innsbruck, Austria). Aliquots were stored at $-20^{\circ} \mathrm{C}$ for determination of protein concentration.

\section{Mitochondrial respiration}

Respiratory oxygen flux was measured by OROBOROS Oxygraph-2k instrument in real-time at $30^{\circ} \mathrm{C}$. Respiration stimulation was determined using substrates for individual mitochondrial complexes I and II. Isolated mitochondria were added into respiratory medium (80 mmol/L KCl, $10 \mathrm{mmol} / \mathrm{L}$ Tris, $3 \mathrm{mmol} / \mathrm{L} \mathrm{MgCl2}, 1$ $\mathrm{mmol} / \mathrm{L}$ EDTA, $5 \mathrm{mmol} / \mathrm{L} \mathrm{KH} 2 \mathrm{PO} 4, \mathrm{pH} 7.4)$ in respective chambers. First, glutamate $(10 \mathrm{mmol} / \mathrm{L})$ and malate ( $2 \mathrm{mmol} / \mathrm{L}$ ) were added (substrates for mitochondrial complex I) and cytochrome C (10 $\mu \mathrm{mol} / \mathrm{L})$ to test outer mitochondrial membrane integrity. Addition of ADP (2 $\mathrm{mmol} / \mathrm{L}$ ) shows inner membrane integrity and coupling of oxidation and phosphorylation. Addition of succinate $(10 \mathrm{mmol} / \mathrm{L})$ indicates mitochondrial complex II function. Combination of ascorbate $(2 \mathrm{mmol} / \mathrm{L})$ and TMPD $(0.5$ $\mathrm{mmol} / \mathrm{L})$ is substrate for cytochrome $\mathrm{C}$ oxidase. Final inhibition with $\mathrm{KCN}$ ( $1 \mathrm{mmol} / \mathrm{L})$ excludes nonspecific oxygen consumption. Index of respiratory control was determined as a ratio between respiration at state III (+ADP) and state IV (-ADP). Oroboros software Datlab 5 version 5.1.0.20 (Oroboros Instruments Corp, Innsbruck, Austria) was used for calculating oxygen uptake rates and for presenting the oxygraphic curve. Oxygen uptake in isolated mitochondria is expressed as $\mathrm{pmol} / \mathrm{s} / \mathrm{mg}$ protein [50]. Presented data are subject of three independent experiments performed in technical duplicates.

\section{Mouse model}

The cell line MDA-MB-231 (Human breast carcinoma, Leibniz Institute DSMZ-German Collection of Microorganisms and Cell Cultures, Braunschweig, Germany) was used for in in vivo experiments. Cells were cultured under following conditions: $37^{\circ} \mathrm{C}, 5 \% \mathrm{CO}_{2}$, Dulbecco's Modified Eagle's Medium (DMEM) with 10\% FBS, 2 mmol/L L-glutamine, and non-essential amino acids $100 \times$ (all Sigma Aldrich, USA).Outbred mouse females (nu/ nu strain Foxn-1) were used for xenograft experiment, with a body weight 20-22 g (AnLab. Ltd., Czech Republic; bred in Centre for Experimental Biomodels, Charles University, Czech Republic). Mice were placed in IVC cages with radiation-sterilized bedding SAWI- Research Bedding (AnLab, Czech Republic) fed with radiation sterilized diet ssniff (AnLab Ltd., Czech Republic) and autoclaved water ad libitum. A lighting regimen was $12 \mathrm{~h}$ light/12 h darkness, temperature $23{ }^{\circ} \mathrm{C}$ and humidity $50-60 \%$.Mice were transplanted with MDA-MB-231cell line $\left(1 \times 10^{7}\right.$ tumor cells/mouse in a $100 \mu \mathrm{l}$ volume together with 50 $\mu$ l of matrigel - Sigma, USA) subcutaneously to the right flank. The application of the test compound was initiated 7 days later. QB was applied by injection into eye in 8 separate dosages (0.2 $\mathrm{mg}$ in DMSO per animal) twice a week. During experiment, sizes of tumors were measured with caliper. The mice were euthanized by overdosing the anesthetics at the end of the experiment tumors were removed and subjected to further analysis.

All animal studies were carried out according to the experimental project approved by Ministry of Education, Youth and Sport of the Czech Republic.

\section{Histology}

Dissected tumours were fixed in $3.8 \%$ paraformaldehyde in PBS, washed in PBS and transferred in $30 \%$ saccharose in PBS. Tumours were embedded into mounting medium (Cryomount) on dry ice and stored at $-80^{\circ} \mathrm{C}$. Cryosections $(12 \mu \mathrm{m})$ were prepared using 
cryostat Leica CM3050 S and stored at $-80^{\circ} \mathrm{C}$. Sections were permeabilized by $0.5 \%$ Triton $\mathrm{X}-100$ in PBS, blocked by $1 \%$ BSA in PBS and stained with rat antimouse F4/80 primary antibody (EXBIO, Prague, Czech Republic), followed by staining with goat anti-rat IgG $(\mathrm{H}+\mathrm{L})$ secondary antibody Alexa Fluor 594 conjugate (Thermo Fisher Scientific, USA). Staining of nuclei and sample mounting was performed by Fluoroshield with DAPI (Sigma-Aldrich). Visualisation was performed using an inverted microscope (Olympus IX71) using 20x objective. Acquisition and data processing was performed using DP Controller 2.2.1.227 software.

\section{Molecular modeling}

Crystal structures of yeast mitochondrial complex I (CI) from Yarrowia lipolytica (PDB ID 4wz7) and porcine mitochondrial complex II (CII) (PDB ID 1zoy) were used for modeling. The program $3 \mathrm{~V}$ was used to identify internal cavities connecting the iron-sulfur clusters with the predicted ubiquinone binding cavity in the CI crystal structure [51]. The geometry of ubiquinone and QB was optimized using DFT-D method with TPSS functional and TZVP basis set $[52,53]$. The effect of water solvation was treated implicitly using COSMO with $\varepsilon=78.4$. All the optimizations were performed in the TurboMole suite of programs. The Python Molecular Viewer (PMV 1.5.6 rc3) was used to set the docking parameters [54]. AutoDock Vina version 1.1.2 was used for ubiquinone and QB docking to CI and CII structures [55]. For the CI structure, the ligands were allowed to sample docking poses in a 60 $\times 60 \times 60 \AA$ box centered around the predicted ubiquinone binding cavity, covering large portion of the lower part of the peripheral arm ( $\mathrm{Q}$ module) and the transmembrane PP module of the membrane arm. For the CII structure the ligands sampled a box of $30 \times 30 \times 30 \AA$ around the known crystal position of the ubiquinone moiety.

\section{ACKNOWLEDGMENTS}

We thank to Karel Harant and Pavel Talacko from Laboratory of Mass Spectrometry, Biocev, Faculty of Science, Charles University, where proteomic analysis has been done.

\section{CONFLICTS OF INTEREST}

Authors disclose no conflicts of interest.

\section{FUNDING}

Czech Science Foundation (project 13-16565S to J.Č. and M.F.) and (project $14-21095 \mathrm{P}$ to K.V.), The Charles University project UNCE (204013/2012 to J.Č. and 204025/2012 to P.N.), European Regional
Development Fund (BIOCEV CZ.1.05/1.1.00/02.0109 to P.N. and Ji.C.), Neuron Foundation (project IMPULS to K.V.), Czech Academy of Sciences (RVO: 61388971 to P.N. and 86652036 to Ji.Č.) and Ministry of Education Youth and Sports of the Czech Republic (LO1509 to D.K. and LQ1604 to P.N.). Access to instrumental and other facilities was supported by EU (Operational Program Prague - Competitiveness project CZ.2.16/3.1.00/24023).

\section{REFERENCES}

1. Warburg O, Wind F, Negelein E. The Metabolism of Tumors in the Body. J Gen Physiol. 1927; 8:519-30.

2. Warburg O. On respiratory impairment in cancer cells. Science. 1956; 124:269-70.

3. Vander Heiden MG, Cantley LC, Thompson CB. Understanding the Warburg effect: the metabolic requirements of cell proliferation. Science. 2009; 324:102933. https://doi.org/10.1126/science.1160809.

4. David CJ, Chen M, Assanah M, Canoll P, Manley JL. HnRNP proteins controlled by c-Myc deregulate pyruvate kinase mRNA splicing in cancer. Nature. 2010; 463:364-8. https://doi.org/10.1038/nature08697.

5. Papandreou I, Cairns RA, Fontana L, Lim AL, Denko NC. HIF-1 mediates adaptation to hypoxia by actively downregulating mitochondrial oxygen consumption. Cell Metab. 2006; 3:187-97. https://doi.org/10.1016/j. cmet.2006.01.012.

6. Wong N, Ojo D, Yan J, Tang D. PKM2 contributes to cancer metabolism. Cancer Lett. 2015; 356:184-91. https://doi. org/10.1016/j.canlet.2014.01.031.

7. Meiser J, Tumanov S, Maddocks O, Labuschagne CF, Athineos D, Van Den Broek N, Mackay GM, Gottlieb E, Blyth K, Vousden K, Kamphorst JJ, Vazquez A. Serine onecarbon catabolism with formate overflow. Sci Adv. 2016; 2:e1601273. https://doi.org/10.1126/sciadv.1601273.

8. Anastasiou $\mathrm{D}, \mathrm{Yu} \mathrm{Y}$, Israelsen $\mathrm{WJ}$, Jiang JK, Boxer MB, Hong BS, Tempel W, Dimov S, Shen M, Jha A, Yang H, Mattaini KR, Metallo CM, et al. Pyruvate kinase M2 activators promote tetramer formation and suppress tumorigenesis. Nat Chem Biol. 2012; 8:839-47. https://doi. org/10.1038/nchembio. 1060 .

9. Birsoy K, Wang T, Chen WW, Freinkman E, Abu-Remaileh M, Sabatini DM. An Essential Role of the Mitochondrial Electron Transport Chain in Cell Proliferation Is to Enable Aspartate Synthesis. Cell. 2015; 162:540-51. https://doi. org/10.1016/j.cell.2015.07.016.

10. Sullivan LB, Gui DY, Hosios AM, Bush LN, Freinkman E, Vander Heiden MG. Supporting Aspartate Biosynthesis Is an Essential Function of Respiration in Proliferating Cells. Cell. 2015; 162:552-63. https://doi.org/10.1016/j. cell.2015.07.017.

11. Wise DR, DeBerardinis RJ, Mancuso A, Sayed N, Zhang XY, Pfeiffer HK, Nissim I, Daikhin E, Yudkoff 
M, McMahon SB, Thompson CB. Myc regulates a transcriptional program that stimulates mitochondrial glutaminolysis and leads to glutamine addiction. Proc Natl Acad Sci U S A. 2008; 105:18782-7. https://doi. org/10.1073/pnas.0810199105.

12. Valis K, Talacko P, Grobarova V, Cerny J, Novak P. Shikonin regulates C-MYC and GLUT1 expression through the MST1-YAP1-TEAD1 axis. Exp Cell Res. 2016; 349:273-81. https://doi.org/10.1016/j.yexcr.2016.10.018.

13. Valis K, Prochazka L, Boura E, Chladova J, Obsil T, Rohlena J, Truksa J, Dong LF, Ralph SJ, Neuzil J. Hippo/ Mst1 stimulates transcription of the proapoptotic mediator NOXA in a FoxO1-dependent manner. Cancer Res. 2011; 71:946-54. https://doi.org/10.1158/0008-5472.CAN-102203.

14. Farber S, Diamond LK. Temporary remissions in acute leukemia in children produced by folic acid antagonist, 4-aminopteroyl-glutamic acid. $\mathrm{N}$ Engl J Med. 1948; 238:787-93. https://doi.org/10.1056/ NEJM194806032382301.

15. Hermanova I, Arruabarrena-Aristorena A, Valis K, Nuskova H, Alberich-Jorda M, Fiser K, Fernandez-Ruiz S, Kavan D, Pecinova A, Niso-Santano M, Zaliova M, Novak P, Houstek $\mathrm{J}$, et al. Pharmacological inhibition of fatty-acid oxidation synergistically enhances the effect of 1-asparaginase in childhood ALL cells. Leukemia. 2016; 30:209-18. https:// doi.org/10.1038/leu.2015.213.

16. Vander Heiden MG. Targeting cancer metabolism: a therapeutic window opens. Nat Rev Drug Discov. 2011; 10:671-84. https://doi.org/10.1038/nrd3504.

17. Klotz LO, Hou X, Jacob C. 1,4-naphthoquinones: from oxidative damage to cellular and inter-cellular signaling. Molecules. 2014; 19:14902-18. https://doi.org/10.3390/ molecules 190914902.

18. Ramirez O, Motta-Mena LB, Cordova A, Estrada A, Li Q, Martinez L, Garza KM. A small library of synthetic di-substituted 1, 4-naphthoquinones induces ROSmediated cell death in murine fibroblasts. PLoS One. 2014; 9:e106828. https://doi.org/10.1371/journal.pone.0106828.

19. Yang JT, Li ZL, Wu JY, Lu FJ, Chen CH. An oxidative stress mechanism of shikonin in human glioma cells. PLoS One. 2014; 9:e94180. https://doi.org/10.1371/journal. pone.0094180.

20. Singh PK, Sarwar M, Maklashina E, Kotlyar V, Rajagukguk S, Tomasiak TM, Cecchini G, Iverson TM. Plasticity of the quinone-binding site of the complex II homolog quinol:fumarate reductase. J Biol Chem. 2013; 288:24293301. https://doi.org/10.1074/jbc.M113.487082.

21. Baran I, Ganea C, Privitera S, Scordino A, Barresi V, Musumeci F, Mocanu MM, Condorelli DF, Ursu I, Grasso R, Gulino M, Garaiman A, Musso N, et al. Detailed analysis of apoptosis and delayed luminescence of human leukemia Jurkat $\mathrm{T}$ cells after proton irradiation and treatments with oxidant agents and flavonoids. Oxid Med Cell Longev. 2012; 2012:498914. https://doi.org/10.1155/2012/498914.
22. Abdelmohsen K, Gerber PA, von Montfort C, Sies H, Klotz LO. Epidermal growth factor receptor is a common mediator of quinone-induced signaling leading to phosphorylation of connexin-43: role of glutathione and tyrosine phosphatases. J Biol Chem. 2003; 278:38360-7. https://doi.org/10.1074/jbc.M306785200.

23. Stodulkova E, Cisarova I, Kolarik M, Chudickova M, Novak P, Man P, Kuzma M, Pavlu B, Cerny J, Flieger M. Biologically active metabolites produced by the basidiomycete Quambalaria cyanescens. PLoS One. 2015; 10:e118913. https://doi.org/10.1371/journal.pone.0118913.

24. Grobarova V, Valis K, Talacko P, Pavlu B, Hernychova L, Novakova J, Stodulkova E, Flieger M, Novak P, Cerny J. Quambalarine B, a Secondary Metabolite from Quambalaria cyanescens with Potential Anticancer Properties. J Nat Prod. 2016; 79:2304-14. https://doi.org/10.1021/acs. jnatprod.6b00362.

25. Guo L, Shestov AA, Worth AJ, Nath K, Nelson DS, Leeper DB, Glickson JD, Blair IA. Inhibition of Mitochondrial Complex II by the Anticancer Agent Lonidamine. J Biol Chem. 2016; 291:42-57. https://doi.org/10.1074/jbc.M115.697516.

26. Banerjee PS, Hart GW, Cho JW. Chemical approaches to study O-GlcNAcylation. Chem Soc Rev. 2013; 42:4345-57. https://doi.org/10.1039/c2cs35412h.

27. Warmoes MO, Locasale JW. Heterogeneity of glycolysis in cancers and therapeutic opportunities. Biochem Pharmacol. 2014; 92:12-21. https://doi.org/10.1016/j.bcp.2014.07.019.

28. Bond MR, Hanover JA. A little sugar goes a long way: the cell biology of O-GlcNAc. J Cell Biol. 2015; 208:869-80. https://doi.org/10.1083/jcb.201501101.

29. Hart GW, Slawson C, Ramirez-Correa G, Lagerlof O. Cross talk between O-GlcNAcylation and phosphorylation: roles in signaling, transcription, and chronic disease. Annu Rev Biochem. 2011; 80:825-58. https://doi.org/10.1146/ annurev-biochem-060608-102511.

30. Chou TY, Dang CV, Hart GW. Glycosylation of the c-Myc transactivation domain. Proc Natl Acad Sci U S A. 1995; 92:4417-21.

31. Kim D, Fiske BP, Birsoy K, Freinkman E, Kami K, Possemato RL, Chudnovsky Y, Pacold ME, Chen WW, Cantor JR, Shelton LM, Gui DY, Kwon M, et al. SHMT2 drives glioma cell survival in ischaemia but imposes a dependence on glycine clearance. Nature. 2015; 520:363-7. https://doi.org/10.1038/nature14363.

32. Gravel SP, Hulea L, Toban N, Birman E, Blouin MJ, Zakikhani M, Zhao Y, Topisirovic I, St-Pierre J, Pollak M. Serine deprivation enhances antineoplastic activity of biguanides. Cancer Res. 2014; 74:7521-33. https://doi. org/10.1158/0008-5472.CAN-14-2643-T.

33. Israelsen WJ, Vander Heiden MG. Pyruvate kinase: Function, regulation and role in cancer. Semin Cell Dev Biol. 2015; 43:43-51. https://doi.org/10.1016/j. semcdb.2015.08.004.

34. Chaneton B, Hillmann P, Zheng L, Martin AC, Maddocks OD, Chokkathukalam A, Coyle JE, Jankevics A, Holding 
FP, Vousden KH, Frezza C, O’Reilly M, Gottlieb E. Serine is a natural ligand and allosteric activator of pyruvate kinase M2. Nature. 2012; 491:458-62. https://doi.org/10.1038/ nature 11540 .

35. Walsh MJ, Brimacombe KR, Anastasiou D, Yu Y, Israelsen WJ, Hong BS, Tempel W, Dimov S, Veith H, Yang H, Kung C, Yen KE, Dang L, et al. ML265: A potent PKM2 activator induces tetramerization and reduces tumor formation and size in a mouse xenograft model. Probe Reports from the NIH Molecular Libraries Program. (Bethesda (MD). National Center for Biotechnology Information (US). 2010.

36. Mattaini KR, Sullivan MR, Vander Heiden MG. The importance of serine metabolism in cancer. J Cell Biol. 2016; 214:249-57. https://doi.org/10.1083/jcb.201604085.

37. Pavlova NN, Thompson CB. The Emerging Hallmarks of Cancer Metabolism. Cell Metab. 2016; 23:27-47. https://doi. org/10.1016/j.cmet.2015.12.006.

38. Dong LF, Low P, Dyason JC, Wang XF, Prochazka L, Witting PK, Freeman R, Swettenham E, Valis K, Liu J, Zobalova R, Turanek J, Spitz DR, et al. Alphatocopheryl succinate induces apoptosis by targeting ubiquinone-binding sites in mitochondrial respiratory complex II. Oncogene. 2008; 27:4324-35. https://doi. org/10.1038/onc.2008.69.

39. Dong LF, Neuzil J. Mitochondria in cancer: why mitochondria are a good target for cancer therapy. Prog Mol Biol Transl Sci. 2014; 127:211-27. https://doi.org/10.1016/ B978-0-12-394625-6.00008-8.

40. Jara JA, Lopez-Munoz R. Metformin and cancer: Between the bioenergetic disturbances and the antifolate activity. Pharmacol Res. 2015; 101:102-8. https://doi.org/10.1016/j. phrs.2015.06.014.

41. Munkley J, Elliott DJ. Hallmarks of glycosylation in cancer. Oncotarget. 2016; 7:35478-89. https://doi.org/10.18632/ oncotarget.8155.

42. Chen J, Xie J, Jiang Z, Wang B, Wang Y, Hu X. Shikonin and its analogs inhibit cancer cell glycolysis by targeting tumor pyruvate kinase-M2. Oncogene. 2011; 30:4297-306. https://doi.org/10.1038/onc.2011.137.

43. Anastasiou D, Poulogiannis G, Asara JM, Boxer MB, Jiang JK, Shen M, Bellinger G, Sasaki AT, Locasale JW, Auld DS, Thomas CJ, Vander Heiden MG, Cantley LC. Inhibition of pyruvate kinase M2 by reactive oxygen species contributes to cellular antioxidant responses. Science. 2011; 334:127883. https://doi.org/10.1126/science.1211485.

44. Tiwari P. Recent Trends in Therapeutic Approaches for Diabetes Management: A Comprehensive Update. J Diabetes Res. 2015; 2015:340838. https://doi. org/10.1155/2015/340838.
45. Livak KJ, Schmittgen TD. Analysis of relative gene expression data using real-time quantitative PCR and the 2(-Delta Delta C(T)) Method. Methods. 2001; 25:402-8. https://doi.org/10.1006/meth.2001.1262.

46. Gottschalk M, Ivanova G, Collins DM, Eustace A, O'Connor R, Brougham DF. Metabolomic studies of human lung carcinoma cell lines using in vitro (1)H NMR of whole cells and cellular extracts. NMR Biomed. 2008; 21:809-19. https://doi.org/10.1002/nbm.1258.

47. Wishart DS, Tzur D, Knox C, Eisner R, Guo AC, Young N, Cheng D, Jewell K, Arndt D, Sawhney S, Fung C, Nikolai L, Lewis M, et al. HMDB: the Human Metabolome Database. Nucleic Acids Res. 2007; 35:D521-6. https://doi. org/10.1093/nar/gk1923.

48. Masuda T, Tomita M, Ishihama Y. Phase transfer surfactantaided trypsin digestion for membrane proteome analysis. J Proteome Res. 2008; 7:731-40. https://doi.org/10.1021/ pr700658q.

49. Cox J, Hein MY, Luber CA, Paron I, Nagaraj N, Mann M. Accurate proteome-wide label-free quantification by delayed normalization and maximal peptide ratio extraction, termed MaxLFQ. Mol Cell Proteomics. 2014; 13:2513-26. https://doi.org/10.1074/mcp. M113.031591.

50. Mezera V, Endlicher R, Kucera O, Sobotka O, Drahota Z, Cervinkova Z. Effects of Epigallocatechin Gallate on Tert-Butyl Hydroperoxide-Induced Mitochondrial Dysfunction in Rat Liver Mitochondria and Hepatocytes. Oxid Med Cell Longev. 2016; 2016:7573131. https://doi. org/10.1155/2016/7573131.

51. Voss NR, Gerstein M. 3V: cavity, channel and cleft volume calculator and extractor. Nucleic Acids Res. 2010; 38:W555-62. https://doi.org/10.1093/nar/gkq395.

52. Cerny J, Jurecka P, Hobza P, Valdes H. Resolution of identity density functional theory augmented with an empirical dispersion term (RI-DFT-D): a promising tool for studying isolated small peptides. J Phys Chem A. 2007; 111:1146-54. https://doi.org/10.1021/jp066504m.

53. Tao J, Perdew JP, Staroverov VN, Scuseria GE. Climbing the density functional ladder: nonempirical metageneralized gradient approximation designed for molecules and solids. Phys Rev Lett. 2003; 91:146401. https://doi. org/10.1103/PhysRevLett.91.146401.

54. Sanner MF, Duncan BS, Carrillo CJ, Olson AJ. Integrating computation and visualization for biomolecular analysis: an example using python and AVS. Pac Symp Biocomput. 1999: 401-12.

55. Trott $\mathrm{O}$, Olson AJ. AutoDock Vina: improving the speed and accuracy of docking with a new scoring function, efficient optimization, and multithreading. J Comput Chem. 2010; 31:455-61. https://doi.org/10.1002/jcc.21334. 